\title{
Polycyclic aromatic hydrocarbons in primary school environments: Levels and potential risks
}

\author{
Marta Oliveira ${ }^{\mathrm{a}, \mathrm{b}}$, Klara Slezakova ${ }^{\mathrm{a}, \mathrm{b}}$, Joana Madureira ${ }^{\mathrm{c}}$, Eduardo de Oliveira Fernandes ${ }^{\mathrm{c}}$, \\ Cristina Delerue-Matos ${ }^{\mathrm{b}}$, Simone Morais ${ }^{\mathrm{b}, *}$, Maria do Carmo Pereira ${ }^{\mathrm{a}, *}$ \\ a LEPABE, Departamento de Engenharia Química, Faculdade de Engenharia, Universidade do Porto, R. Dr. Roberto Frias, $4200-465$ Porto, Portugal \\ b REQUIMTE-LAQV, Instituto Superior de Engenharia do Porto, Instituto Politécnico do Porto, R. Dr. António Bernardino de Almeida 431, 4200-072 Porto, Portugal \\ c Institute of Science and Innovation on Mechanical Engineering and Industrial Management, Rua Dr. Roberto Frias, 4200-465 Porto, Portugal
}

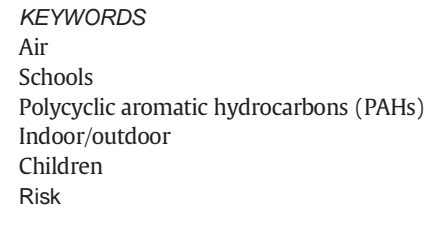

GR A P H I C A L A B S T R A C T

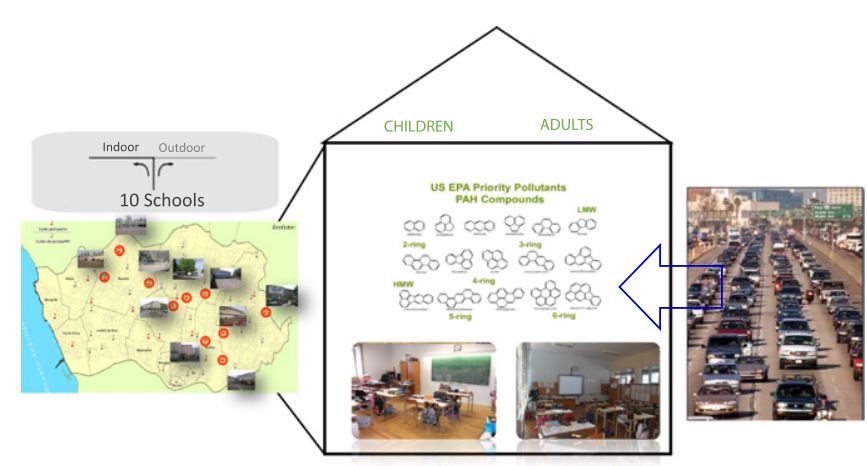

\begin{abstract}
A B S T R A C T
Although polycyclic aromatic hydrocarbons (PAHs) are priority air pollutants that strongly affect human health, information concerning the indoor exposures is still limited. This study characterized PAH levels in primary schools and evaluated risk for the respective students (aged 8-10 years) in comparison with school personnel. During January-April 2014, eighteen particulate-bound $\left(\mathrm{PM}_{2.5}\right)$ PAHs (16 USEPA priority compounds, dibenzo[a,l]pyrene, benzo[j]fluoranthene) were collected (indoors and outdoors) at ten primary urban schools in Portugal. Total mean concentrations $\left(\Sigma_{\mathrm{PAHs}}\right)$ ranged $2.8-54 \mathrm{ng} \mathrm{m}^{-3}$ in indoor air, whereas corresponding outdoor levels were $7.1-48 \mathrm{ng} \mathrm{m}^{-3}$. Indoor/outdoor ratios of lighter congeners (2-3 aromatic rings) demonstrated a contribution from indoor origin while heavier PAHs (4-6 aromatic rings) originated mostly from infiltration of ambient air indoors; traffic (both from diesel and gasoline fuelled vehicles) was the predominant source of indoor PAHs. Total cancer risk of 8-10 years old children exceeded (up to 22 times) USEPA recommended guideline of $10^{-6}$, and 7-87 times WHO health-based threshold of $10^{-5}$. Risk due to indoor exposure in schools was 2-10 times higher than outdoors, mainly because of the higher amount of time that students spent indoors.
\end{abstract}

(c) 2016 Elsevier B.V. All rights reserved.

\footnotetext{
* Corresponding authors.

E-mail addresses: sbm@isep.ipp.pt (S. Morais), mcsp@fe.up.pt (M. do Carmo Pereira).
}

\section{Introduction}

During the last three decades, ambient air pollution and its negative impact on human health has been the major focus of the scientific 
community. The knowledge of the health associated impacts on human health as a result of indoor air pollution has been limited. Indoor air exposure is a complex subject and its comprehension, and subsequently, the establishment and implementation of respective guidelines in order to assure safe indoor environments, are the key focuses of many international organisations. In that regard, the World Health Organization (WHO) has designated a list of priority health-relevant indoor air pollutants (WHO, 2010) including polycyclic aromatic hydrocarbons (PAHs).

PAHs are a class of organic compounds composed of multiple aromatic rings (Haritash and Kaushik, 2009) that are ubiquitously found in the environment. In air, PAHs are distributed between gas phase and particulate matter (Ma et al., 2011; Vasilakos et al., 2007); the majority of particulate PAHs is though bound to fine fraction $\left(\mathrm{PM}_{2.5}\right.$; i.e. aerodynamic diameter below $2.5 \mu \mathrm{m}$ ) (Slezakova et al., 2013a,b). PAHs are produced by incomplete combustion and the major anthropogenic sources include traffic, coal combustion processes, emissions from power plants and waste treatment, and from biomass and wood burning (Hanedar et al., 2014; Ravindra et al., 2008; Samburova et al., 2016; Sarigiannis et al., 2015; Slezakova et al., 2013a,b). Indoor PAHs result from activities such as smoking and cooking, from various fuels and candle burning, as well as from penetration of outdoor emissions (by ventilation systems, due to structural cracks, etc.) (Chen et al., 2012; Kim et al., 2011; Masih et al., 2010; Qi et al., 2014; Shen et al., 2012; Wu et al., 2015). PAHs affect organisms through various toxic actions and because of their mutagenic and potentially carcinogenic properties, the U.S. Environmental Protection Agency (USEPA) classified sixteen PAHs as priority pollutants (USEPA, 2005). Out of these, benzo[a]pyrene has been designated as a human carcinogen (group 1) (IARC, 2010); when assessing cancer risk in humans it has been commonly used as a marker for the carcinogenic PAHs (WHO, 2010). In addition, some individual PAHs are considered as persistent organic pollutants (WHO, 2013) and several PAHs are regarded as endocrine disrupting chemicals (WHO, 2013).

In a view of health consequences, exposure to PAHs is particularly relevant for children (Annesi-Maesano et al., 2007; Bae et al., 2010; Tuntawiroon et al., 2010) who are one of the most susceptible subgroups of the population (Burtscher and Schüepp, 2012; Foos et al., 2008; Singh and Gupta, 2016). Compared to adults, children exposures to airborne pollution are larger because of their faster inhalations, increased number of physical activities, thus allowing for larger intake of toxic compounds (Gilliland, 2009; Pohl and Abadin, 2008). Furthermore, being exposed to genotoxic carcinogenic compounds at a young age may cause various genetic disruptions (such as mutations, sister chromatid exchanges, etc.) (Merlo et al., 2007; Neri et al., 2006; Wigle et al., 2007) resulting in an elevated risk of cancer in the adult life (Carpente and Bushkin-Bedient, 2013; Vedham et al., 2015). Children spend much of their daily time in schools, kindergartens, which raises the scientific interest in understanding the air pollution in these types of indoor microenvironments. Because of the respective health impact, information concerning PAHs in schools has been slowly emerging but the current data is still limited. Data available for primary schools (i.e. children $6-11$ years old) come from a few European studies (Alves et al., 2014; Carpente and Bushkin-Bedient, 2013; Cirillo et al., 2006; Jovanović et al., 2014; Krugly et al., 2014; Moshammer and Neuberger, 2003; Romagnoli et al., 2014), Asia (Jyethi et al., 2014; Ruchirawat et al., 2006, 2007; Tuntawiroon et al., 2007) and USA (Eiguren-Fernandez et al., 2007). However, the majority of those were typically conducted in a limited number of schools (typically 1-2 schools; only two known studies used a greater number of schools, namely 5 and 6; Krugly et al., 2014; Romagnoli et al., 2014), often situated just in one/same type of environment. In addition, information regarding the risk assessments due to exposure to PAHs in primary schools is scarce (Krugly et al., 2014) with slightly more evidence available for preschool children (i.e. aged between three and five years) (Oliveira et al., 2015, 2016; Wilson et al., 2003).

This study investigates the concentrations of 16 USEPA priority PAHs and dibenzo[a,l]pyrene and benzo[j]fluoranthene at ten primary schools representative of different urban environments (city centre, residential, industrial). The indoor $\mathrm{PAH}$ profiles were characterized and the influence of PAHs in ambient air on their presence indoors was evaluated. Analysis of diagnostic ratios was conducted to identify potential sources of PAHs. Moreover, toxicity equivalency factors (TEF) and USEPA risk approach method (USEPA, 2016) were used for the chronic carcinogenic risk assessment of the subpopulations of students (8-10 years) and of school personnel.

\section{Material and methods}

\subsection{Schools characterization}

Eighteen particulate-bound priority PAHs (naphthalene, acenaphthylene, acenaphthene fluorene, phenanthrene, anthracene, fluoranthene, pyrene, benz[ $a$ ]anthracene, chrysene, benzo[ $b]$ fluoranthene, benzo[ $k]$ fluoranthene, benzo[ $a]$ pyrene, dibenz[ $(a, h]$ anthracene, benzo[g,h,i]perylene, indeno[1,2,3-cd]pyrene, dibenzo[ $a, l]$ pyrene, and benzo[j]fluoranthene) were sampled during ten consecutive weeks (January-April 2014) that corresponded to period of the 2nd trimester at ten primary public schools (S1-S10) in Oporto Metropolitan Area (north of Portugal; 2nd largest metropolitan area in the country). Emissions from vehicular traffic, a power plant, an incineration unit, and an oil refinery with a petrochemical complex situated near the international shipping port are the major pollution sources of the respective areas (Pereira et al., 2007; Slezakova et al., 2013a,b). The schools (Fig. 1) were selected in order to evaluate different urban environments (city centre, residential, industrial). Detailed information concerning the characterized schools, namely age and size of buildings (year of construction, size, etc.), their usage, atypical or visible occurrences and construction problems, potential indoor sources (including types of heating systems), and the descriptions of each school outdoor area (including potential emission sources) are summarized in Table 1. No calculations of ventilation rates were possible due to the limited information obtained. S2 was extensively ventilated at the beginning of the day, whereas other classrooms were ventilated when necessary. Schools (S2-S4, S6, S8 and S10) ventilated their classrooms for a few minutes during recesses/breaks, whereas other schools (S4, S8 and S10) ventilated their classrooms during class times.

\subsection{Sampling}

At each school particulate samples were collected continuously (for periods of $24 \mathrm{~h}$ ) during three consecutive week days (Tuesday-Thursday), avoiding Mondays and Fridays (children daily schedules were often irregular). The sample collection was conducted in classrooms where children spent the majority of time. Two rooms (8-10 years old children) per each school were simultaneously investigated, resulting in a total of 20 classrooms. In total 85 samples were obtained. Classroom characteristics (room area, height), used materials (windows, furniture, and etc.), information about cleaning and maintenance were collected, as well as the details regarding any equipment (such as printers, copy machines, type of blackboards, vegetation), or use of environmental modifiers (namely insecticides or air fresheners). The main characteristics of studied rooms are shown in Table 1.

The indoor $\mathrm{PM}_{2.5}$ were sampled according to USEPA IP-10A method (USEPA, 1990) Samples were collected daily using a personal environmental monitor ( $\mathrm{PEM}^{\mathrm{TM}}$; SKC Ltd., Dorset, UK), i.e. single stage impactor $\left(\mathrm{PM}_{2.5}\right.$ ) that was combined with personal air sampling pump (AirChek ${ }^{\circledR}$ 2000; SKC Ltd., Dorset, UK); an air flow rate of $2.0 \mathrm{~L} \mathrm{~min}^{-1}$ was used. The pumps were daily calibrated according to the manufacturer's instructions with the flow being verified at the end of sampling. Without compromising the usual use of the rooms, the impactors' inlets were positioned at least $1.0 \mathrm{~m}$ above the floor and at least $1 \mathrm{~m}$ from any obstacle (including walls), away from room entrances or windows. Safety 


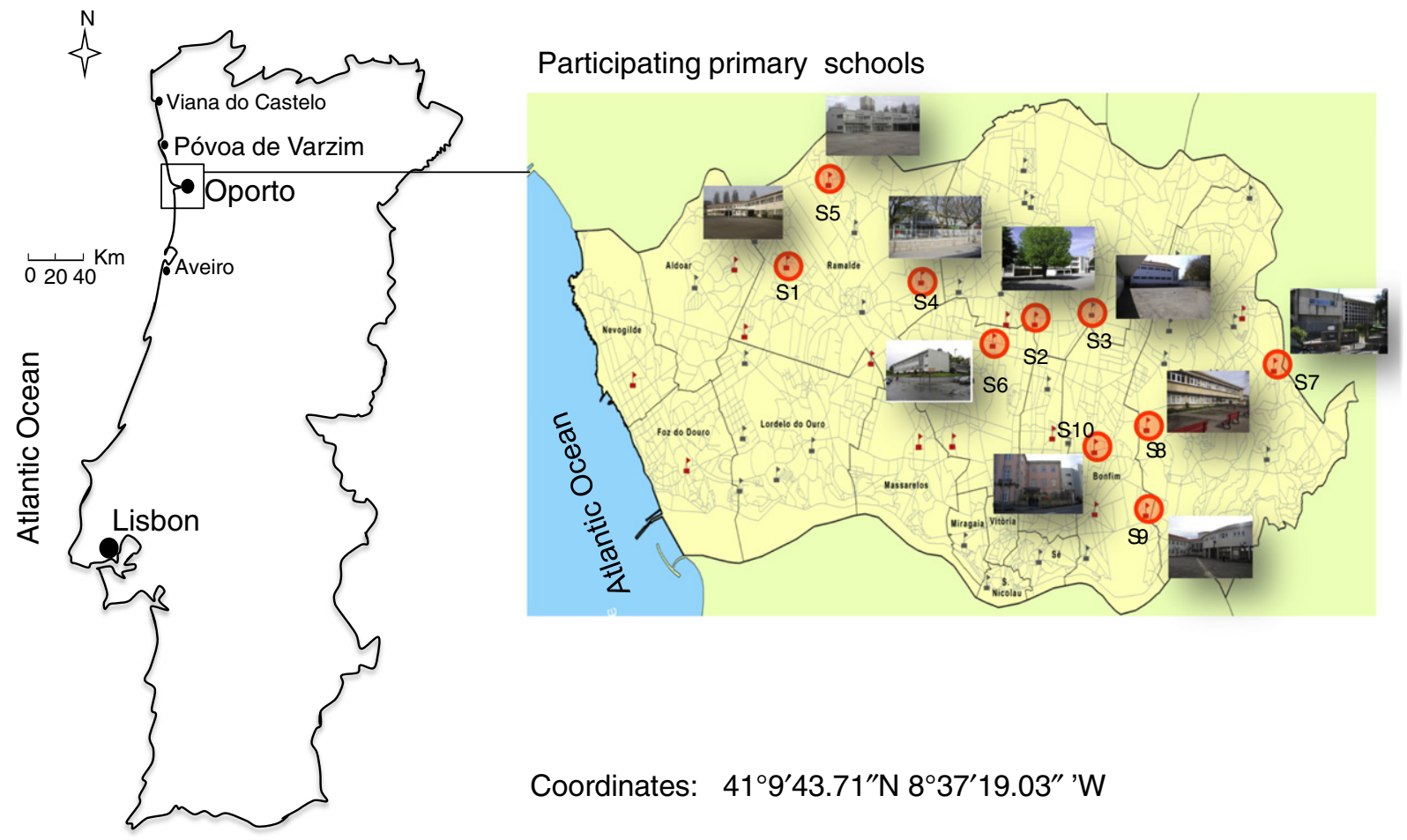

Fig. 1. Geographic locations of the characterized ten primary schools.

measures were taken in order to maintain adequate environments for the respective students.

Simultaneously with indoor monitoring, particulate-bound PAHs were collected outdoors (i.e. in ambient air) except at S1, S3 and S4 because of the school outdoor dispositions and/or safety reasons. Outdoor $\mathrm{PM}_{2.5}$-bound PAHs were daily sampled in school yards with apparatuses situated in open zones (away from trees and vegetation, fences and any other obstacles that could hinder the sample collection). Sampling apparatuses consisted of constant low-flow $\left(2.3 \mathrm{~m}^{3} \mathrm{~h}^{-1}\right)$ pumps (model Bravo H2; TCR TECORA, Paris, France) with $\mathrm{PM}_{2.5}$ sampling head (in accordance with norm EN14907).

Polytetrafluoroethylene membrane disks ( $2 \mu \mathrm{m}$ porosity, Ø37 and $47 \mathrm{~mm}$ for indoor and outdoor air, respectively; SKC Ltd., Dorset, UK) were used for the collection of PM samples. $\mathrm{PM}_{2.5}$ were determined gravimetrically as previously described (Slezakova et al., 2011a, 2014). After the gravimetric mass determinations, filters were placed in polyethylene containers and stored (at $-20^{\circ} \mathrm{C}$ ) for the chemical analysis.

Relative humidity and temperature were recorded with an IAQCALC monitor (model 7545, TSI Inc., Shoreview, MN) with logging intervals of $5 \mathrm{~min}$.

During the whole sampling period, information regarding type, duration and frequency of ventilation, number of occupants in the respective classrooms, and existence of any potential source or relevant indoor activities was registered every day. In addition, teachers and school personnel were questioned on a daily basis in order to obtain further information about unusual activities and/or occurrences.

\subsection{PAHs quantification}

The PAHs extraction and quantification for particulate samples were accomplished using previously validated method (Castro et al., 2009, 2011). Briefly, microwave-assisted extraction (MAE) and liquid chromatography with photodiode array (PAD) (for acenaphthylene) and fluorescence detection (for all the other compounds) were applied. Recoveries varied from $81.4 \pm 8.8 \%$ to $112.0 \pm 1.1 \%$, for all PAHs except for naphthalene $(62.3 \pm 18.0 \%)$ and anthracene $(67.3 \pm 5.7 \%)$. Limits of detection (LODs) corresponding to outdoor sampling ranged from $1 \mathrm{pg}$ $\mathrm{m}^{-3}$ (for anthracene, benzo[k]fluoranthene, chrysene, benz[ $a$ ]anthracene, phenanthrene and indeno[1,2,3-cd]pyrene) to 148 $\mathrm{pg} \mathrm{m}^{-3}$ (for acenaphthylene), while the respective quantification limits (LOQs) were 3.4-492 $\mathrm{pg} \mathrm{m}^{-3}$. For indoor air samples, LODs ranged between $3 \mathrm{pg} \mathrm{m}^{-3}$ for anthracene, benzo[k]fluoranthene, chrysene, benz[ $a$ anthracene, phenanthrene and indeno[1,2,3-cd]pyrene) and $565 \mathrm{pg} \mathrm{m}^{-3}$ (for acenaphthylene), whereas the respective LOQs were 10-1886 $\mathrm{pg} \mathrm{m}^{-3}$. Standards were analysed daily, as well as blank MAE extracts (from filter blank), between samples to verify instrumental performance. Each quantification was performed in triplicate.

\subsection{Risk assessment}

Toxicity equivalency factors (TEF) according to Nisbet and LaGoy (Boström et al., 2002) were used for the calculations of TEF adjusted concentrations. Subsequently, the lifetime lung cancer risk was calculated according to WHO methodology (WHO, 1987, 2000).

The PAHs carcinogenic risk (TR) was estimated based on the procedure indicated by USEPA (2016). TR was assessed as the incremental probability of a subject to develop cancer, over a lifetime, as a consequence of exposure to that potential carcinogen (USEPA, 1989). The methodology for the risk assessment (including the respective equations) are described in detailed in Slezakova et al. (2014). Type of conducted activities by children were registered at each school (Table $1 \mathrm{~S}$ of the Supplementary material). Table $2 S$ presents an example of TR calculation; default parameters for the TR calculations are presented in Tables $3 \mathrm{~S}$ and $4 \mathrm{~S}$. For comparison, target carcinogenic risk was also estimated for school personnel considering the adults with the same daily schedules as the children; 15 years of exposure duration (i.e. employment) was used.

\section{Results and discussion}

\section{1. $P M_{2.5}$ and PAH levels}

The concentrations of $\mathrm{PM}_{2.5}$ indoors ranged from $4.5 \mu \mathrm{g} \mathrm{m} \mathrm{m}^{-3}$ (minimum) to $85 \mu \mathrm{g} \mathrm{m}^{-3}$ (maximum), whereas the indoor means of each 
Table 1

Characterization of ten studied primary schools ( $\mathrm{S} 1-\mathrm{S} 10)$ ( $\mathrm{n}=20$ classrooms).

\begin{tabular}{|c|c|c|c|c|c|c|c|c|c|c|}
\hline & S1 & S2 & S3 & S4 & S5 & S6 & S7 & S8 & S9 & S10 \\
\hline Location & $\begin{array}{l}\text { Residential area with } \\
\text { industrial influence }\end{array}$ & City centre & City centre & City centre & $\begin{array}{l}\text { Residential area, } \\
\text { industrial influence }\end{array}$ & City centre & $\begin{array}{l}\text { Residential area } \\
\text { surrounded with } \\
\text { greens }\end{array}$ & City centre & Residential area & $\begin{array}{l}\text { City } \\
\text { centre-background } \\
\text { area }\end{array}$ \\
\hline Site characterization & Urban-industrial & Urban-traffic & Urban-traffic & Urban-traffic & Urban-industrial & Urban-traffic & Urban-background & Urban-traffic & Urban-traffic & Urban-traffic \\
\hline Year of construction & 1968 & 1938 & 1959 & 1959 & 1980 & 1958 & 1963 & 1957 & 1982 & 1950 \\
\hline $\begin{array}{l}\text { Year of } \\
\text { refurbishment }\end{array}$ & 2012 & 2011 & 2007 & 2010 & 2008 & 2004 & 2005 & 2004 & 2008 & 2005 \\
\hline $\begin{array}{l}\text { Outdoor emissions } \\
\text { sources }\end{array}$ & $\begin{array}{l}\text { Local traffic; Car } \\
\text { park; Industrial site } \\
\text { within } 10 \mathrm{~km}\end{array}$ & $\begin{array}{l}\text { Local traffic; Car } \\
\text { park; Gasoline } \\
\text { station }\end{array}$ & Local traffic & $\begin{array}{l}\text { Local traffic Car } \\
\text { park; Gasoline } \\
\text { station }\end{array}$ & $\begin{array}{l}\text { Local traffic Car } \\
\text { park; Industrial site } \\
\text { within } 10 \mathrm{~km}\end{array}$ & $\begin{array}{l}\text { Local traffic; Car } \\
\text { park }\end{array}$ & $\begin{array}{l}\text { Local traffic Car } \\
\text { park; Gasoline } \\
\text { station }\end{array}$ & $\begin{array}{l}\text { Local traffic; Car } \\
\text { park }\end{array}$ & $\begin{array}{l}\text { Local traffic; Car } \\
\text { park }\end{array}$ & Local traffic \\
\hline $\begin{array}{l}\text { Number of students } \\
\text { (6-11 years old })\end{array}$ & 256 & 446 & 452 & 442 & 386 & 394 & 370 & 288 & 264 & 420 \\
\hline $\begin{array}{l}\text { Classroom area } \\
\left(\mathrm{m}^{2}\right)\end{array}$ & $48.9 \pm 0.0$ & $50.3 \pm 5.4$ & $46.2 \pm 0.0$ & $46.8 \pm 0.0$ & $56.6 \pm 0.2$ & $59.2 \pm 23.5$ & $50.1 \pm 0.9$ & $50.4 \pm 0.1$ & $56.16 \pm 0.08$ & $50.6 \pm 5.1$ \\
\hline $\begin{array}{l}\text { Classroom height } \\
\left(\mathrm{m}^{2}\right)\end{array}$ & $3.50 \pm 0.0$ & $3.2 \pm 0.2$ & $3.4 \pm 0.0$ & $3.4 \pm 0.0$ & $3.3 \pm 0.3$ & $3.3 \pm 0.0$ & $3.4 \pm 0.0$ & $3.0 \pm 0.1$ & $3.3 \pm 0.0$ & $3.6 \pm 0.0$ \\
\hline $\begin{array}{l}\text { Classroom } \\
\text { occupancy } \\
\text { density ( } \mathrm{m}^{2} \text { per } \\
\text { occupant) }\end{array}$ & $2.7 \pm 0.2$ & $2.6 \pm 0.3$ & $2.0 \pm 0.3$ & $2.1 \pm 0.2$ & $2.9 \pm 0.4$ & $2.95 \pm 0.9$ & $3.2 \pm 0.6$ & $2.0 \pm 0.1$ & $2.4 \pm 0.2$ & $2.3 \pm 0.5$ \\
\hline Furniture & Plywood furniture & Plywood furniture & Wood furniture & Wood furniture & Wood furniture & Wood furniture & $\begin{array}{l}\text { Wood and metal } \\
\text { furniture }\end{array}$ & $\begin{array}{l}\text { Wood and metal } \\
\text { furniture }\end{array}$ & Wood furniture & Wood furniture \\
\hline Windows & $\begin{array}{l}\text { Aluminium frame, } \\
\text { double glazed }\end{array}$ & $\begin{array}{l}\text { Metal frame, singe } \\
\text { glazed }\end{array}$ & $\begin{array}{l}\text { Aluminium frame, } \\
\text { single glazed }\end{array}$ & $\begin{array}{l}\text { Aluminium frame, } \\
\text { single glazed }\end{array}$ & $\begin{array}{l}\text { Aluminium frame, } \\
\text { double glazed }\end{array}$ & $\begin{array}{l}\text { Aluminium frame, } \\
\text { double glazed }\end{array}$ & $\begin{array}{l}\text { Aluminium frame, } \\
\text { single glazed }\end{array}$ & $\begin{array}{l}\text { Aluminium frame, } \\
\text { single glazed }\end{array}$ & $\begin{array}{l}\text { Aluminium frame, } \\
\text { single glazed }\end{array}$ & $\begin{array}{l}\text { Aluminium frame, } \\
\text { double glazed }\end{array}$ \\
\hline Heating units & $\begin{array}{l}\text { Hot water } \\
\text { radiators/convectors }\end{array}$ & $\begin{array}{l}\text { Electrical } \\
\text { radiators/convectors }\end{array}$ & $\begin{array}{l}\text { Electrical } \\
\text { radiators/convectors }\end{array}$ & $\begin{array}{l}\text { Electrical } \\
\text { radiators/convectors }\end{array}$ & $\begin{array}{l}\text { Electrical } \\
\text { radiators/convectors }\end{array}$ & $\begin{array}{l}\text { Electrical } \\
\text { radiators/convectors }\end{array}$ & $\begin{array}{l}\text { Electrical } \\
\text { radiators/convectors }\end{array}$ & $\begin{array}{l}\text { Electrical } \\
\text { radiators/convectors }\end{array}$ & $\begin{array}{l}\text { Electrical } \\
\text { radiators/convectors }\end{array}$ & $\begin{array}{l}\text { Electrical } \\
\text { radiators/convectors }\end{array}$ \\
\hline Ventilation & & & & & & & & & & \\
\hline Beginning of day & no & yes & no & no & no & no & no & no & no & no \\
\hline During classes & no & no & no & yes & no & no & no & yes & no & yes \\
\hline During brakes & no & yes & yes & yes & no & yes & no & yes & no & yes \\
\hline After classes & no & no & no & no & no & no & no & no & no & no \\
\hline During cleaning & no & yes & yes & yes & yes & yes & yes & yes & yes & yes \\
\hline Cleaning schedule & $\begin{array}{l}\text { Twice per day } \\
\text { (beginning and end } \\
\text { of day) }\end{array}$ & $\begin{array}{l}\text { Twice (beginning } \\
\text { and end of day) }\end{array}$ & $\begin{array}{l}\text { Once per day (end } \\
\text { of day) }\end{array}$ & $\begin{array}{l}\text { Once (beginning of } \\
\text { day) }\end{array}$ & $\begin{array}{l}\text { Once per day (end of } \\
\text { day) }\end{array}$ & $\begin{array}{l}\text { Twice per day } \\
\text { (beginning and end } \\
\text { of day) }\end{array}$ & $\begin{array}{l}\text { Once per day } \\
\text { (beginning of day) }\end{array}$ & $\begin{array}{l}\text { Once per day (end } \\
\text { of day) }\end{array}$ & $\begin{array}{l}\text { Once per day (end } \\
\text { of day) }\end{array}$ & $\begin{array}{l}\text { Once per day (end } \\
\text { of day) }\end{array}$ \\
\hline $\begin{array}{l}\text { Number of copy } \\
\text { machine }\end{array}$ & 1 & 1 & 1 & 3 & 1 & 1 & 1 & 1 & 1 & 2 \\
\hline Other & & & & & \multicolumn{6}{|c|}{$\begin{array}{l}\text { Meals cooked at } \\
\text { school1159M. Oliveiraetal./ScienceoftheTotalEnvironment575(2017)1156-1167 }\end{array}$} \\
\hline
\end{tabular}


school were between 9.2 and $66 \mu \mathrm{g} \mathrm{m}^{-3}$. Levels in outdoor air ranged from 2.4 to $67 \mu \mathrm{g} \mathrm{m}^{-3}, \mathrm{PM}_{2.5}$ means in ambient air for each school were between 5.3 and $34 \mu \mathrm{g} \mathrm{m}^{-3}$. The highest $\mathrm{PM}_{2.5}$ mean $(25-67 \mu \mathrm{g}$ $\mathrm{m}^{-3}$ ) in outdoor air were observed at school S2, possibly reflecting seasonal influences (Finardi et al., 2015) and/or local source of vehicle emissions (i.e. parking lot situated nearby). The lowest median concentrations measured were at $\mathrm{S} 9\left(8.7 \pm 3.6 \mu \mathrm{g} \mathrm{m}^{-3}\right)$ and $\mathrm{S} 105.3 \pm 2.3 \mu \mathrm{g}$ $\mathrm{m}^{-3}$ ) and were situated in a residential and suburban areas, respectively.
The concentrations of indoor $\mathrm{PM}_{2.5}-\mathrm{PAHs}$ in the ten studied primary schools are summarized in Table 2, which reports the indoor average levels (as well as the ranges). Total levels of $\mathrm{EPAHs}$ indoors ranged from 1.7-60 ng $\mathrm{m}^{-3}$ whereas the school mean concentrations ranged from 2.8 to $54 \mathrm{ng} \mathrm{m}^{-3}$. Among all the schools, the highest $\Sigma_{\text {PAHs }}$ were observed at S2 (Table 2) being approximately 3-7 times higher than in S1-S8; these levels were 10 and 19 times higher than at S10 and S9, respectively. There were no specific indoor emission sources or activities of occupants that could justify the levels of PAHs at this school;

Table 2

Indoor levels of $\mathrm{PM}_{2.5}$-bound PAHs at ten studied schools S1-S10 $\left(\mathrm{ng} \mathrm{m}^{-3}\right)(\mathrm{n}=60)$.

\begin{tabular}{|c|c|c|c|c|c|c|c|c|c|c|}
\hline & $\mathrm{S} 1$ & S2 & S3 & $\mathrm{S} 4$ & S5 & S6 & S7 & S8 & S9 & S10 \\
\hline Naph & $2.1(0.80-3.5)$ & $1.2(0.88-1.5)$ & $\begin{array}{l}0.39 \\
(0.36-0.41)\end{array}$ & $\begin{array}{l}0.21 \\
(0.18-0.25)\end{array}$ & $\begin{array}{l}0.11 \\
(0.10-0.12)\end{array}$ & $\begin{array}{l}0.22 \\
(0.18-0.27)\end{array}$ & $\begin{array}{l}0.27 \\
(0.20-0.33)\end{array}$ & $\begin{array}{l}0.28 \\
(0.14-0.43)\end{array}$ & $\begin{array}{l}0.17(8.6 \times \\
\left.10^{-2}-0.26\right)\end{array}$ & $\begin{array}{l}0.26 \\
(0.19-0.34)\end{array}$ \\
\hline Acy & $11(11-12)$ & $16(10-21)$ & $6.6(5.8-7.3)$ & $5.4(4.5-6.4)$ & $4.7(3.7-5.6)$ & $7.9(6.5-9.4)$ & $3.9(1.8-6.0)$ & $4.4(3.2-5.7)$ & $\begin{array}{l}0.88 \\
(0.40 \times-1.8)\end{array}$ & $\begin{array}{l}0.42 \\
(0.40-0.45)\end{array}$ \\
\hline Ace & $1.5(1.3-1.8)$ & $2.3(2.3-2.4)$ & $\begin{array}{l}0.91 \\
(0.71-1.1)\end{array}$ & $1.2(0.81-1.6)$ & $\begin{array}{l}0.91 \\
(0.79-1.0)\end{array}$ & $1.2(1.1-1.4)$ & $1.3(1.1-1.5)$ & $\begin{array}{l}1.1 \\
(0.93-1.2)\end{array}$ & $1.1(0.89-1.1)$ & $1.2(0.71-1.6)$ \\
\hline Flu & $\begin{array}{l}6.7 \times 10^{-2} \\
(5.0 \times \\
10^{-2}-8.6 \times \\
\left.10^{-2}\right)\end{array}$ & $\begin{array}{l}8.3 \times 10^{-2} \\
(7.3 \times \\
10^{-2}-9.6 \times \\
\left.10^{-2}\right)\end{array}$ & $\begin{array}{l}1.8 \times 10^{-2} \\
(1.7 \times \\
10^{-2}-2.0 \times \\
\left.10^{-2}\right)\end{array}$ & $\begin{array}{l}1.2 \times 10^{-2} \\
(1.0 \times \\
10^{-2}-1.3 \times \\
\left.10^{-2}\right)\end{array}$ & $\begin{array}{l}1.6 \times 10^{-2} \\
(1.3 \times \\
10^{-2}-1.9 \times \\
\left.10^{-2}\right)\end{array}$ & $\begin{array}{l}4.1 \times 10^{-2} \\
(2.6 \times \\
10^{-2}-5.6 \times \\
\left.10^{-2}\right)\end{array}$ & $\begin{array}{l}3.1 \times 10^{-2} \\
(2.7 \times \\
10^{-2}-3.6 \times \\
\left.10^{-2}\right)\end{array}$ & $\begin{array}{l}2.1 \times 10^{-2} \\
(2.0 \times \\
10^{-2}-2.4 \times \\
\left.10^{-2}\right)\end{array}$ & $\begin{array}{l}5.9 \times 10^{-3} \\
(3.2 \times \\
10^{-3}-1.1 \times \\
\left.10^{-2}\right)\end{array}$ & $\begin{array}{l}1.8 \times 10^{-2} \\
(1.6 \times \\
10^{-2}-2.1 \times \\
\left.10^{-2}\right)\end{array}$ \\
\hline Phe & $\begin{array}{l}0.37 \\
(0.33-0.42)\end{array}$ & $\begin{array}{l}0.87 \\
(0.72-1.0)\end{array}$ & $\begin{array}{l}0.17 \\
(0.15-0.19)\end{array}$ & $\begin{array}{l}0.10(9.5 \times \\
\left.10^{-2}-0.11\right)\end{array}$ & $\begin{array}{l}0.22 \\
(0.18-0.25)\end{array}$ & $\begin{array}{l}0.24 \\
(0.23-0.27)\end{array}$ & $\begin{array}{l}0.24 \\
(0.18-0.29)\end{array}$ & $\begin{array}{l}0.25 \\
(0.23-0.27)\end{array}$ & $\begin{array}{l}8.6 \times 10^{-2} \\
(7.9 \times \\
10^{-2}-9.5 \times \\
\left.10^{-2}\right)\end{array}$ & $\begin{array}{l}0.26 \\
(0.25-0.28)\end{array}$ \\
\hline Ant & $\begin{array}{l}7.5 \times 10^{-2} \\
(7.2 \times \\
10^{-2}-7.8 \times \\
\left.10^{-2}\right)\end{array}$ & $\begin{array}{l}1.7 \times 10^{-2} \\
(7.4 \times \\
10^{-2} \cdot-2.9 \times \\
\left.10^{-2}\right)\end{array}$ & $\begin{array}{l}1.5 \times 10^{-2} \\
(5.3 \times \\
10^{-2}-2.7 \times \\
\left.10^{-2}\right)\end{array}$ & $\begin{array}{l}5.4 \times 10^{-3} \\
(3.0 \times \\
10^{-3}-6.7 \times \\
\left.10^{-3}\right)\end{array}$ & $\begin{array}{l}8.1 \times 10^{-2} \\
\left(3.0 \times 10^{-3}-\right. \\
\left.1.0 \times 10^{-2}\right)\end{array}$ & $\begin{array}{l}1.8 \times 10^{-2} \\
(1.7 \times \\
10^{-2}-2.0 \times \\
\left.10^{-2}\right)\end{array}$ & $\begin{array}{l}3.0 \times 10^{-3} \\
(3.0 \times \\
10^{-3}-3.0 \times \\
\left.10^{-3}\right)\end{array}$ & $\begin{array}{l}1.1 \times 10^{-2} \\
(6.7 \times \\
10^{-3}-1.5 \times \\
\left.10^{-2}\right)\end{array}$ & $\begin{array}{l}4.3 \times 10^{-3} \\
(3.0 \times \\
10^{-3}-4.7 \times \\
\left.10^{-3}\right)\end{array}$ & $\begin{array}{l}5.2 \times 10^{-3} \\
(3.0 \times \\
10^{-3} .-6.5 \times \\
\left.10^{-3}\right)\end{array}$ \\
\hline Fln & $\begin{array}{l}0.29(2.8 \times \\
\left.10^{-2}-0.55\right)\end{array}$ & $6.1(4.8-7.5)$ & $\begin{array}{l}0.11(1.6 \times \\
\left.10^{-2}-0.22\right)\end{array}$ & $\begin{array}{l}7.5 \times 10^{-3} \\
(6.4 \times \\
10^{-3}-2.3 \times \\
\left.10^{-2}\right)\end{array}$ & $\begin{array}{l}0.54 \\
(0.11-1.1)\end{array}$ & $\begin{array}{l}4.5 \times 10^{-2} \\
(2.5 \times \\
10^{-2}-6.8 \times \\
\left.10^{-2}\right)\end{array}$ & $\begin{array}{l}3.3 \times 10^{-2} \\
(2.1 \times \\
10^{-2}-4.7 \times \\
\left.10^{-2}\right)\end{array}$ & $\begin{array}{l}0.57(5.0 \times \\
\left.10^{-2}-1.1\right)\end{array}$ & $\begin{array}{l}1.9 \times 10^{-2} \\
(1.4 \times \\
10^{-2}-2.5 \times \\
\left.10^{-2}\right)\end{array}$ & $\begin{array}{l}3.0 \times 10^{-2} \\
(2.0 \times \\
10^{-2}-4.0 \times \\
\left.10^{-2}\right)\end{array}$ \\
\hline Pyr & $\begin{array}{l}0.20 \\
(0.18-0.21)\end{array}$ & $1.4(1.1-1.7)$ & $\begin{array}{l}0.21 \\
(0.17-0.26)\end{array}$ & $\begin{array}{l}5.8 \times 10^{-2} \\
(5.4 \times \\
10^{-2}-6.4 \times \\
\left.10^{-2}\right)\end{array}$ & $\begin{array}{l}0.27 \\
(0.24-0.29)\end{array}$ & $\begin{array}{l}0.28 \\
(0.25-0.31)\end{array}$ & $\begin{array}{l}0.53 \\
(0.27-0.77)\end{array}$ & $\begin{array}{l}0.31 \\
(0.23-0.39)\end{array}$ & $\begin{array}{l}4.7 \times 10^{-2} \\
(4.5 \times \\
10^{-2}-4.9 \times \\
\left.10^{-2}\right)\end{array}$ & $\begin{array}{l}0.14(9.9 \times \\
\left.10^{-2}-0.17\right)\end{array}$ \\
\hline $\mathrm{B}[a] \mathrm{A}$ & $\begin{array}{l}4.6 \times 10^{-2} \\
(4.14 \times \\
10^{-2}-5.10 \times \\
\left.10^{-2}\right)\end{array}$ & $\begin{array}{l}0.67 \\
(0.46-0.90)\end{array}$ & $\begin{array}{l}9.3 \times 10^{-2} \\
(8.02 \times \\
\left.10^{-2}-0.11\right)\end{array}$ & $\begin{array}{l}1.2 \times 10^{-2} \\
(1.00 \times \\
10^{-2}-1.53 \times \\
\left.10^{-2}\right)\end{array}$ & $\begin{array}{l}7.4 \times 10^{-2} \\
(6.14 \times \\
10^{-2}-9.25 \times \\
\left.10^{-2}\right)\end{array}$ & $\begin{array}{l}7.4 \times 10^{-2} \\
(6.76 \times \\
10^{-2}-8.09 \times \\
\left.10^{-2}\right)\end{array}$ & $\begin{array}{l}0.16(8.23 \times \\
\left.10^{-2}-0.239\right)\end{array}$ & $\begin{array}{l}7.5 \times 10^{-2} \\
(6.22 \times \\
\left.10^{-2}-0.878\right)\end{array}$ & $\begin{array}{l}1.3 \times 10^{-2} \\
(1.14 \times \\
10^{-2}-1.55 \times \\
\left.10^{-2}\right)\end{array}$ & $\begin{array}{l}4.8 \times 10^{-2} \\
(3.84 \times \\
10^{-2}-5.75 \times \\
\left.10^{-2}\right)\end{array}$ \\
\hline Chry & $\begin{array}{l}0.13 \\
(0.11-0.15)\end{array}$ & $1.3(0.89-1.8)$ & $\begin{array}{l}0.18 \\
(0.17-0.19)\end{array}$ & $\begin{array}{l}3.4 \times 10^{-2} \\
(3.2 \times \\
10^{-2}-3.8 \times \\
\left.10^{-2}\right)\end{array}$ & $\begin{array}{l}0.17 \\
(0.13-0.20)\end{array}$ & $\begin{array}{l}0.17 \\
(0.15-0.18)\end{array}$ & $\begin{array}{l}0.38 \\
(0.19-0.61)\end{array}$ & $\begin{array}{l}0.20 \\
(0.18-0.21)\end{array}$ & $\begin{array}{l}3.6 \times 10^{-2} \\
(3.0 \times \\
10^{-2}-4.1 \times \\
\left.10^{-2}\right)\end{array}$ & $\begin{array}{l}0.11(7.5 \times \\
\left.10^{-2}-0.13\right)\end{array}$ \\
\hline $\mathrm{B}[b+j] \mathrm{F}$ & $\begin{array}{l}0.70 \\
(0.59-0.83)\end{array}$ & $6.6(5.2-8.1)$ & $\begin{array}{l}0.83 \\
(0.80-0.87)\end{array}$ & $\begin{array}{l}0.16 \\
(0.15-0.16)\end{array}$ & $\begin{array}{l}0.97 \\
(0.85-1.1)\end{array}$ & $\begin{array}{l}0.57 \\
(0.55-0.60)\end{array}$ & $\begin{array}{l}1.9 \\
(0.93-2.83)\end{array}$ & $\begin{array}{l}1.1 \\
(0.85-1.3)\end{array}$ & $\begin{array}{l}0.11(9.4 \times \\
\left.10^{-2}-0.13\right)\end{array}$ & $\begin{array}{l}0.56 \\
(0.55-0.57)\end{array}$ \\
\hline $\mathrm{B}[k] \mathrm{F}$ & $\begin{array}{l}0.12 \\
(0.10-0.15)\end{array}$ & $1.32(1.0-1.6)$ & $\begin{array}{l}0.16 \\
(0.15-0.16)\end{array}$ & $\begin{array}{l}3.1 \times 10^{-2} \\
(3.0 \times \\
10^{-2}-3.3 \times \\
\left.10^{-2}\right)\end{array}$ & $\begin{array}{l}0.19 \\
(0.18-0.21)\end{array}$ & $\begin{array}{l}0.11(9.7 \times \\
\left.10^{-2}-0.12\right)\end{array}$ & $\begin{array}{l}0.34 \\
(0.17-0.51)\end{array}$ & $\begin{array}{l}0.19 \\
(0.15-0.24)\end{array}$ & $\begin{array}{l}2.0 \times 10^{-2} \\
(1.6 \times \\
10^{-2}-2.4 \times \\
\left.10^{-2}\right)\end{array}$ & $\begin{array}{l}8.9 \times 10^{-2} \\
(7.1 \times \\
\left.10^{-2}-0.11\right)\end{array}$ \\
\hline $\mathrm{B}[a] \mathrm{P}$ & $\begin{array}{l}0.27 \\
(0.21-0.33)\end{array}$ & $3.5(2.9-4.2)$ & $\begin{array}{l}0.38 \\
(0.35-0.46)\end{array}$ & $\begin{array}{l}6.0 \times 10^{-2} \\
(5.6 \times \\
10^{-2}-6.4 \times \\
\left.10^{-2}\right)\end{array}$ & $\begin{array}{l}0.57 \\
(0.55-0.58)\end{array}$ & $\begin{array}{l}0.22 \\
(0.19-0.26)\end{array}$ & $\begin{array}{l}0.77 \\
(0.31-1.3)\end{array}$ & $\begin{array}{l}0.40 \\
(0.31-0.48)\end{array}$ & $\begin{array}{l}3.7 \times 10^{-2} \\
(3.1 \times \\
10^{-2}-4.4 \times \\
\left.10^{-2}\right)\end{array}$ & $\begin{array}{l}0.20 \\
(0.19-0.22)\end{array}$ \\
\hline $\mathrm{D}[a, h] \mathrm{A}$ & $1.5(1.3-1.8)$ & 5.0 (n.d.-10) & $1.4(1.3-1.5)$ & $\begin{array}{l}0.37 \\
(0.36-0.39)\end{array}$ & $2.3(2.1-2.3)$ & $1.1(0.98-1.1)$ & $4.1(2.1-6.2)$ & $1.9(1.4-2.5)$ & $\begin{array}{l}0.16 \\
(0.14-0.18)\end{array}$ & $1.2(1.1-1.4)$ \\
\hline $\mathrm{B}[$ ghi $] \mathrm{P}$ & $\begin{array}{l}0.61 \\
(0.51-0.74)\end{array}$ & $4.1(3.5-4.6)$ & $\begin{array}{l}0.61 \\
(0.56-0.66)\end{array}$ & $\begin{array}{l}0.24 \\
(0.22-0.25)\end{array}$ & $\begin{array}{l}0.86 \\
(0.80-0.91)\end{array}$ & $\begin{array}{l}0.47 \\
(0.46-0.48)\end{array}$ & $\begin{array}{l}1.3 \\
(0.67-1.9)\end{array}$ & $\begin{array}{l}0.78 \\
(0.71-0.86)\end{array}$ & $\begin{array}{l}0.15 \\
(0.14-0.17)\end{array}$ & $\begin{array}{l}0.46 \\
(0.43-0.49)\end{array}$ \\
\hline InP & $\begin{array}{l}0.44 \\
(0.36-0.52)\end{array}$ & $4.0(3.2-4.7)$ & $\begin{array}{l}0.52 \\
(0.48-0.56)\end{array}$ & $\begin{array}{l}0.12 \\
(0.11-0.12)\end{array}$ & $\begin{array}{l}0.75 \\
(0.69-0.83)\end{array}$ & $\begin{array}{l}0.26 \\
(0.22-0.29)\end{array}$ & $\begin{array}{l}1.3 \\
(0.61-1.9)\end{array}$ & $\begin{array}{l}0.60 \\
(0.38-0.85)\end{array}$ & $\begin{array}{l}5.7 \times 10^{-2} \\
(4.8 \times \\
10^{-2}-6.4 \times \\
\left.10^{-2}\right)\end{array}$ & $\begin{array}{l}0.33 \\
(0.30-0.36)\end{array}$ \\
\hline$\Sigma_{\mathrm{PAHs}}$ & $20(18-22)$ & $54(48-60)$ & $13(12-13)$ & $8.0(7.4-8.6)$ & $13(11-14)$ & $13(11-15)$ & $16(9.2-24)$ & $12(9.2-15)$ & $2.8(1.7-4.0)$ & $5.3(5.0-5.6)$ \\
\hline$\Sigma_{\text {PAHscarc. }}$ & $5.4(3.5-7.3)$ & $24(15-33)$ & $4.0(3.8-4.2)$ & $1.0(0.96-1.0)$ & $5.1(5.0-5.2)$ & $2.7(2.5-2.9)$ & $9.2(4.7-14)$ & $4.8(3.8-5.8)$ & $\begin{array}{l}0.61 \\
(0.47-0.75)\end{array}$ & $2.8(2.6-3.0)$ \\
\hline
\end{tabular}

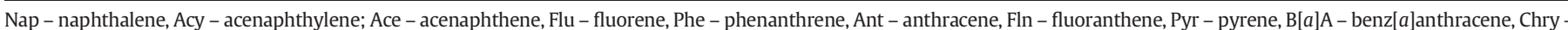

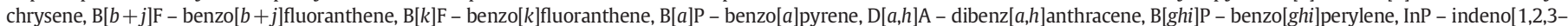
cd]pyrene;

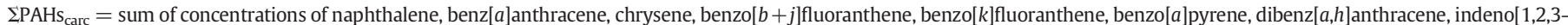
cd]pyrene;

Note: The concentrations of Ant (at S7) and Acy (at S9) were below the LOD, thus the value of the respective LOD $/ \sqrt{2}$ was used (Hornung and Reed, 1990);

Dibenzo[a,l]pyrene $<$ LOD in $100 \%$ of collected samples and hence not included. 
however, S2 was the only school that used heating systems during the sampling. In addition, S2 was the only school equipped with metal framed windows ( single glazed; Table 1). It must be though highlighted that the highest levels of PAHs in ambient air were also observed at S2 (Table 3), at the same time S2 was the only school with classrooms being intensively ventilated at the beginning of the day (directly before classes started; Table 1). Therefore, the higher indoor levels might result from the combination of various factors, namely from occupants' indoor activities, characteristics of the buildings/classrooms, and from infiltration of outdoor particles to indoor air (by ventilations, due to inferior building isolation, etc.) as was similarly previously reported (Amato et al., 2014; Moreno et al., 2014; Rivas et al., 2014, 2015). On the contrary, the minimum indoor levels of PAHs were observed at S9 which was situated in a residential area.

Overall, the data on PAHs in educational environments is rather scarce. In addition, the available studies were conducted with different approaches, variation of the considered PAH congeners, and often PAHs analysed in different PM fractions (mainly in coarse fraction, or even unspecified); all of these further complicate the comparisons between the reported findings. As for data available for $\mathrm{PM}_{2.5}$-bound PAHs, Eiguren-Fernandez et al. (2007) reported total concentrations of 15 compounds in a range of $0.4-1.8 \mathrm{ng} \mathrm{m}^{-3}$ in indoor air of schools in Southern California. Analyzing the same 15 PAHs, Krugly et al. (2014) found much higher levels of $\mathrm{PM}_{2.5}$-bound PAHs (20.1-131 $\mathrm{ng} \mathrm{m}^{-3}$ ) in indoor air of schools in Lithuania. With the exception to S2, the indoor levels in schools of this study $\left(\Sigma_{\text {PAHs }} 2.8-20 \mathrm{ng} \mathrm{m}^{-3}\right)$ were rather similar to schools in Rome (1.6-16 $\mathrm{ng} \mathrm{m}^{-3}$ ) (Cirillo et al., 2006). Romagnoli et al. (2014) also found $\mathrm{PM}_{2.5}$-bound PAHs in similar ranges (1.8-8.3 ng $\mathrm{m}^{-3}$ ) in indoor air of schools (sampled during winter season).

The compositional profiles between the ten characterized schools in this study were relatively similar. In majority of the schools (S1-S6, S8) acenaphthylene was the most (or the second most abundant at S7 and S9) indoor particulate-bound $\mathrm{PAH}$. On average this compound accounted for $40 \%$ of total particulate content ( $\left.\Sigma_{\mathrm{PAHs}}\right)$, at each school its contribution ranged between $29 \%$ (at S2) up to 68\% (S4) of $\Sigma_{\text {PAHs }}$. The second most abundant compound was dibenz $[a, h]$ anthracene which was in agreement with the previous studies in the respective area (Castro et al., 2011; Oliveira et al., 2015). This PAH approximately accounted for $13 \% \Sigma_{\text {PAHs }}$ (range 5-25\% of $\Sigma_{\text {PAHs }}$ at S4 and S7, respectively) and its high abundance suggests emissions from vehicular traffic (light-duty gasoline; Ravindra et al., 2008). Acenapthene and benzo[ $b+$ j]fluoranthene were the other abundant PAHs, accounting for 12\% (4$22 \%$ at S2 and S10) and 7\% (2-11\% at S7 and S4) of $\Sigma_{\text {PAHs }}$; the contributions of the remaining compounds were much lower (i.e. $<4 \%$ ).

The indoor variation of particulate-bound $\mathrm{EPAHs}$ was not statistically significant ( $p>0.05$ applying the nonparametric Mann - Whitney $U$ test), except for S7, where PAHs from 1 classroom exhibited significantly lower levels. This variation was attributed to different position/distance of the classrooms from the main street.

WHO recommendation for naphthalene (defined as annual guideline value of $10 \mu \mathrm{g} \mathrm{m}^{-3}$; WHO, 2010) is the only existing guidelines for PAHs in indoor air. The maximum levels of naphthalene $(2.1 \pm 1.5$

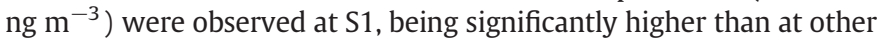
schools $(p<0.05)$. However, this mean (as well as other schools) was still below the recommended guideline. It is necessary to point out that naphthalene is a volatile PAH, predominantly found in gas phase (Krugly et al., 2014; Oliveira et al., 2016). Therefore, the assessment of PAHs vapour phase in school environments should be conducted in future in order to correctly evaluate levels of this compound.

Ten individual compounds, namely naphthalene, benz[a]anthracene, chrysene, benzo[b]fluoranthene, benzo[j]fluoranthene, benzo[ $k]$ luoranthene, benzo[a]pyrene, dibenzo[ $[a, l]$ pyrene, dibenz $[a, h]$ anthracene and indeno[1,2,3-cd]pyrene were designated by International Agency Research on Cancer as possible or probable carcinogens (IARC, 2002, 2010). The levels of these carcinogenic PAHs (i.e. $\Sigma_{\text {PAHscarc }}$ ) at the indoor air of the studied schools are

Table 3

Outdoor levels of $\mathrm{PM}_{2.5}$-bound PAHs at studied schools $\left(\mathrm{ng} \mathrm{m}^{-3}\right)(\mathrm{n}=25)$.

\begin{tabular}{|c|c|c|c|c|c|c|c|}
\hline & S2 & S5 & S6 & S7 & S8 & S9 & $\mathrm{S} 10$ \\
\hline Naph & $0.34(0.17-0.53)$ & $\begin{array}{l}9.8 \times 10^{-2}(6.3 \times \\
\left.10^{-2}-0.13\right)\end{array}$ & $\begin{array}{l}9.8 \times 10^{-2}(8.2 \times \\
\left.10^{-2}-0.12\right)\end{array}$ & $\begin{array}{l}0.16(4.1 \times \\
\left.10^{-2}-0.25\right)\end{array}$ & $0.16(0.15-0.17)$ & $\begin{array}{l}0.11(2.6 \times \\
\left.10^{-2}-0.21\right)\end{array}$ & $\begin{array}{l}3.8 \times 10^{-2}(2.5 \times \\
\left.10^{-2}-5.2 \times 10^{-2}\right)\end{array}$ \\
\hline Acy & $3.9(3.48-4.18)$ & $2.2(1.20-2.95)$ & $2.2(2.04-2.52)$ & $3.6(0.477-6.23)$ & $0.96(0.682-1.17)$ & $2.2(1.08-3.23)$ & $0.79(0.172-1.37)$ \\
\hline Ace & $0.97(0.69-1.2)$ & $0.38(0.27-0.45)$ & $0.36(0.27-0.52)$ & $1.15(0.88-1.4)$ & $0.35(0.29-0.42)$ & $0.49(0.33-0.72)$ & $0.39(0.29-0.53)$ \\
\hline Flu & $\begin{array}{l}9.7 \times 10^{-2}(8.5 \times \\
\left.10^{-2}-0.11\right)\end{array}$ & $\begin{array}{l}3.4 \times 10^{-2}(1.6 \times \\
\left.10^{-2}-0.07\right)\end{array}$ & $\begin{array}{l}1.8 \times 10^{-2}(1.7 \times \\
\left.10^{-2}-2.0 \times 10^{-2}\right)\end{array}$ & $\begin{array}{l}2.9 \times 10^{-2}(2.2 \times \\
\left.10^{-2}-3.5 \times 10^{-2}\right)\end{array}$ & $\begin{array}{l}3.0 \times 10^{-2}(9.6 \times \\
\left.10^{-3}-4.8 \times 10^{-2}\right)\end{array}$ & $\begin{array}{l}2.3 \times 10^{-2}(1.4 \times \\
\left.10^{-2}-3.3 \times 10^{-2}\right)\end{array}$ & $\begin{array}{l}1.2 \times 10^{-2}(9.7 \times \\
\left.10^{-3}-1.6 \times 10^{-2}\right)\end{array}$ \\
\hline Phe & $0.71(0.47-0.97)$ & $0.25(0.15-0.43)$ & $0.16(0.15-0.18)$ & $0.49(0.46-0.53)$ & $0.35(0.15-0.64)$ & $0.20(0.11-0.39)$ & $\begin{array}{l}0.12(4.0 \times \\
\left.10^{-2}-0.21\right)\end{array}$ \\
\hline Ant & $\begin{array}{l}2.1 \times 10^{-2}(1.9 \times \\
\left.10^{-2}-2.2 \times 10^{-2}\right)\end{array}$ & $\begin{array}{l}5.9 \times 10^{-3}(4.7 \times \\
\left.10^{-3}-7.3 \times 10^{-3}\right)\end{array}$ & $\begin{array}{l}5.7 \times 10^{-3}(3.8 \times \\
\left.10^{-3}-7.6 \times 10^{-2}\right)\end{array}$ & $\begin{array}{l}3.7 \times 10^{-2}(2.2 \times \\
\left.10^{-2}-6.4 \times 10^{-2}\right)\end{array}$ & $\begin{array}{l}2.3 \times 10^{-2}(5.1 \times \\
\left.10^{-3}-6.5 \times 10^{-2}\right)\end{array}$ & $\begin{array}{l}8.1 \times 10^{-3}(6.9 \times \\
\left.10^{-3}-1.6 \times 10^{-2}\right)\end{array}$ & $\begin{array}{l}2.2 \times 10^{-3}(1.4 \times \\
\left.10^{-3}-2.9 \times 10^{-3}\right)\end{array}$ \\
\hline Fln & $4.8(0.29-9.4)$ & $\begin{array}{l}2.0 \times 10^{-2}(2.0 \times \\
\left.10^{-2}-2.1 \times 10^{-2}\right)\end{array}$ & $\begin{array}{l}2.1 \times 10^{-2}(1.7 \times \\
\left.10^{-2}-2.5 \times 10^{-2}\right)\end{array}$ & $0.26(0.16-0.34)$ & $1.3\left(2.4 \times 10^{-2}-3.3\right)$ & $\begin{array}{l}0.13(1.7 \times \\
\left.10^{-2}-0.38\right)\end{array}$ & $\begin{array}{l}2.4 \times 10^{-2}(2.2 \times \\
\left.10^{-2}-2.5 \times 10^{-2}\right)\end{array}$ \\
\hline Pyr & $2.2(1.9-2.4)$ & $0.59(0.18-1.4)$ & $0.30(0.20-0.43)$ & $0.77(0.71-0.82)$ & $0.54(0.18-0.88)$ & $0.49(0.17-1.1)$ & $0.34(0.27-0.34)$ \\
\hline $\mathrm{B}[a] \mathrm{A}$ & $1.4(0.95-1.9)$ & $\begin{array}{l}0.415(3.7 \times \\
\left.10^{-2}-1.2\right)\end{array}$ & $\begin{array}{l}7.95 \times 10^{-2}(3.7 \times \\
\left.10^{-2}-0.12\right)\end{array}$ & $0.184(0.17-0.20)$ & $\begin{array}{l}0.321(5.4 \times \\
\left.10^{-2}-0.64\right)\end{array}$ & $\begin{array}{l}0.239(5.2 \times \\
\left.10^{-2}-0.45\right)\end{array}$ & $\begin{array}{l}0.118(8.4 \times \\
\left.10^{-2}-0.18\right)\end{array}$ \\
\hline Chry & $2.7(1.8-3.7)$ & $\begin{array}{l}0.81(9.3 \times \\
\left.10^{-2}-2.2\right)\end{array}$ & $0.22(0.11-0.35)$ & $0.42(0.37-0.48)$ & $0.76(0.13-1.3)$ & $0.57(0.13-1.1)$ & $0.27(0.18-0.39)$ \\
\hline $\mathrm{B}[b+j] \mathrm{F}$ & $7.6(5.3-10)$ & $2.1(0.31-5.5)$ & $0.85(0.39-1.5)$ & $1.3(1.0-1.7)$ & $3.1(0.63-5.6)$ & $1.8(0.40-3.3)$ & $0.97(0.79-1.3)$ \\
\hline $\mathrm{B}[k] \mathrm{F}$ & $1.6(1.1-2.1)$ & $\begin{array}{l}0.43(5.9 \times \\
\left.10^{-2}-1.1\right)\end{array}$ & $\begin{array}{l}0.17(7.6 \times \\
\left.10^{-2}-0.31\right)\end{array}$ & $0.24(0.19-0.32)$ & $0.56(0.11-1.0)$ & $\begin{array}{l}0.34(7.6 \times \\
\left.10^{-2}-0.67\right)\end{array}$ & $0.19(0.16-0.25)$ \\
\hline $\mathrm{B}[a] \mathrm{P}$ & $3.6(2.3-4.8)$ & $1.1\left(9.4 \times 10^{-2}-3.3\right)$ & $0.31(0.15-0.53)$ & $0.47(0.43-0.52)$ & $1.2(0.17-2.2)$ & $\begin{array}{l}0.63(9.1 \times \\
\left.10^{-2}-1.5\right)\end{array}$ & $0.33(0.30-0.38)$ \\
\hline $\mathrm{D}[a, h] \mathrm{A}$ & $11(7.7-15)$ & $3.3(0.57-7.9)$ & $1.4(0.59-2.5)$ & $2.3(1.8-3.2)$ & $4.2(1.1-6.8)$ & $2.4(0.65-5.1)$ & $1.7(1.4-2.2)$ \\
\hline $\mathrm{B}[$ ghi $] \mathrm{P}$ & $3.5(2.8-4.3)$ & $1.0(0.20-2.5)$ & $0.45(0.21-0.756)$ & $0.71(0.56-0.96)$ & $1.1(0.36-2.3)$ & $0.77(0.23-1.5)$ & $0.54(0.46-0.64)$ \\
\hline InP & $3.8(2.6-5.0)$ & $1.1(0.15-2.7)$ & $0.42(0.17-0.81)$ & $0.70(0.56-1.0)$ & $1.4(0.33-2.4)$ & $0.77(0.18-1.7)$ & $0.5(0.46-0.64)$ \\
\hline$\Sigma_{\text {PAHs }}$ & $48(33-63)$ & $14(6.7-32)$ & $7.1(4.7-10)$ & $13(12-15)$ & $16(15-27)$ & $11(3.7-20)$ & $6.4(5.0-8.4)$ \\
\hline$\Sigma_{\text {PAHscarc. }}$ & $32(22-42)$ & $9.3(1.4-24)$ & $3.6(1.6-6.2)$ & $5.7(4.6-7.7)$ & $12(2.7-20)$ & $6.8(1.6-14)$ & $4.2(3.4-5.3)$ \\
\hline
\end{tabular}

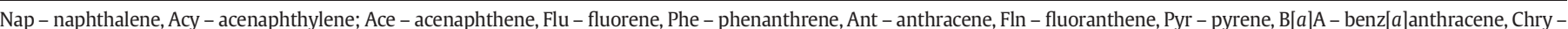

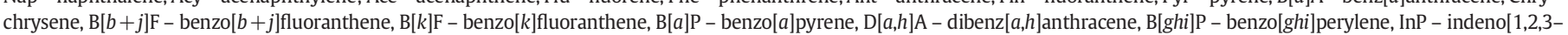
cd]pyrene;

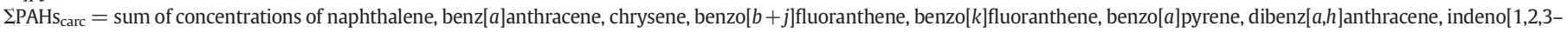
cd]pyrene;

Dibenzo[a,l]pyrene $<$ LOD in $100 \%$ of collected samples and hence not reported. 
also presented in Table 2. In general, the levels in Table 2 were in similar concentration ranges to those in schools in Rome during winter (Romagnoli et al., 2014; mean $\Sigma_{\text {PAHscarc }}$ of 1.0-6.8 $\mathrm{ng} \mathrm{m}^{-3}$ ); however, for spring season these authors found much lower concentrations (0.21-0.71 $\left.\mathrm{ng} \mathrm{m}^{-3}\right)$. In this study, $\Sigma_{\text {PAHscarc }}$ accounted for approximately $35 \%$ of $\Sigma_{\text {PAHs }}$ (range between $12 \%$ at S4 to $56 \%$ at S7). Among the carcinogenic PAHs, dibenz $[a, h]$ anthracene exhibited the highest contribution at all schools accounting for approximately $36 \%$ of $\Sigma_{\text {PAHscarc }}$ (between $21 \%$ at S2 and $45 \%$ at S7). In a view of potential health implications, it is necessary to remark that at all studied schools dibenz $[a, h]$ anthracene ranked among the abundant compounds even when all the detected PAHs were considered. Other major carcinogens were: benzo[ $b+j]$ fluoranthene, that on average accounted for $23 \%$ of $\Sigma_{\text {PAHscarc }}$ (range of $13-28 \%$ at S1 and S2, respectively), and benzo[a]pyrene (mean: $9 \%$ of $\Sigma_{\text {PAHscarc }}$; range: $5-15 \%$ of $\Sigma_{\text {PAHscarc }}$ at S1 and S2, respectively).

The levels of PAHs monitored in ambient air of schools are presented in Table 3. Total levels of $\Sigma_{\text {PAHs }}$ outdoors ranged from 3.7-63 $\mathrm{ng} \mathrm{m}^{-3}$. With the exception to S2 (range: 33-63 $\mathrm{ng} \mathrm{m}^{-3}$; mean of $48 \mathrm{ng} \mathrm{m}^{-3}$ ), mean concentrations of $\mathrm{PM}_{2.5}$-bound PAHs in outdoor air of the Portuguese primary schools ( $6.4 \mathrm{ng} \mathrm{m}^{-3}$ at $\mathrm{S} 10-16 \mathrm{ng} \mathrm{m}^{-3}$ at S8) were similar to the levels reported in winter for ambient air in Rome (4.5-18 ng $\mathrm{m}^{-3}$; obtained from fixed stations of the environmental monitoring network; Gatto et al., 2013) or for outdoors of schools in Rome (6.3$9.5 \mathrm{ng} \mathrm{m}^{-3}$; Romagnoli et al., 2014). For warmer seasons (0.21-1.3 ng $\mathrm{m}^{-3}$ in ambient air by Gatto et al., 2013; $0.42-1.7 \mathrm{ng} \mathrm{m}^{-3}$ reported by Romagnoli et al., 2014) and in other European countries (Lithuania; 41-120 ng m ${ }^{-3}$; Krugly et al., 2014) considerably different levels of particulate-bound PAHs were observed. Although there was no specific emission source that could justify the increased levels of ambient $\mathrm{PAHs}$ at $\mathrm{S} 2$, the respective sampling was conducted during a period with harsh meteorological conditions (causing atmospheric inversion), which could lead in overall elevated levels of pollutants in the atmosphere. In that regard, it is necessary to point out that levels of PAHs in air are seasonally dependent (with higher levels typically observed in winter; Finardi et al., 2015; Jedynska et al., 2014) due to a different impact of residential heating emissions (absent in summer), variation of PAHs degradation due to light and oxidants availability during summer, and/or due to the atmospheric dispersion efficiency. In agreement, Finardi et al. (2015) reported levels of benzo[a]pyrene of $3.0 \mathrm{ng} \mathrm{m}^{-3}$ in winter in outdoor air of dwellings (schools, homes, offices) in Rome whereas it was $<0.1 \mathrm{ng} \mathrm{m}^{-3}$ for the summer season. Using benzo[a]pyrene as indicator of carcinogenic PAHs, the current European legislation on ambient air (Directive 2004/107/EC, 2005) sets annual target value of $1 \mathrm{ng} \mathrm{m}^{-3}$ for carcinogenic PAHs in $\mathrm{PM}_{10}$ (particulate matter with aerodynamic diameter below $10 \mu \mathrm{m}$ ). The mean concentration of this PAH (averaged of all outdoor data) was $0.94 \pm 1.18 \mathrm{ng} \mathrm{m}^{-3}$, with the $30 \%$ of measurements exceeding the annual limit value. Nevertheless, it is necessary to emphasize that these findings need to be implicated carefully as the sampling period did not include whole calendar year (and all seasons); Finardi et al. (2015) previously demonstrated the need of continuous monitoring of atmospheric PAH once the use of discontinuous measurements (as currently designated in EU Directive 2004/107/EC, 2005) does not guarantee a reliable evaluation of seasonal levels (Finardi et al., 2015).

The results in Tables 2-3 also show that total PAH concentrations in outdoor vs. indoor air of each schools were not statistically different $(p<0.05)$ with the exception to the S6 where indoor $\Sigma_{\mathrm{PAHs}}$ was approximately twice higher than outdoors. School S6 was the only one equipped with kitchen. Unlike the other schools, meals were directly cooked at the school premises which could result in overall higher indoor levels (in comparison to outdoors). In addition, emissions from some other indoor sources could also contribute to indoor levels of PAHs. The observed potential sources were: use of heating systems, use of cleaning agents, and use of artistic supplies for children activities (such as solvents, adhesives, paints, wax and candles, etc.). PAHs compositional profiles in ambient air were similar among the schools and showed some similarities to indoors. Once again dibenz[ $a, h]$ anthracene (mean of $23 \%$ of $\Sigma_{\text {PAHs }} ; 18-28 \%$ at S7 and S10), acenaphthylene (mean of $17 \%$ of $\Sigma_{\text {PAHs }} ; 3-32 \%$ at S8 and S6) and benzo $[b+j]$ fluoranthene (15\% of $\Sigma_{\text {PAHs }}$; ranging between $10 \%$ at $S 7$ and $20 \%$ at S8) were among the most abundant compounds. Lastly, on average $\Sigma_{\text {PAHscarc }}$ comprised outdoors $54 \%$ of $\Sigma_{\text {PAHs }}$ (range $45-74 \%$ at S7 and S8, respectively).

\subsection{Source analysis}

\subsubsection{Indoor-to-outdoor ratios}

Analysis of PAHs indoor-to-outdoor concentration ratios ( $\mathrm{I} / \mathrm{O}) \mathrm{can}$ allow deeper understanding of the potential impact of indoor and/or outdoor emissions on indoors. The calculated I/O ratios particulate PAHs at studied primary schools are shown in Fig. 2. At all schools, light PAHs with 2 aromatic rings (namely naphthalene, acenapthene, acenaphthylene) exhibited I/O much higher than unity, thus suggesting potential contribution of indoor sources. These findings were consistent with other studies. I/O > > 1 were observed for semi-volatile PAHs in indoor air of 5 primary Lithuanian schools (Krugly et al., 2014) which could be explained by evaporation from used construction materials and occupants' activities. Emission sources observed during the sampling period included the use of heating systems (electrical convertors), utilization of artistic materials (wax melting, paints, solvents, clays, etc.) and/or the use of various cleaning agents. In addition, some children celebrated their birthdays in the classrooms (cakes with lighted candles) which might lead to elevated PAHs. These results were in agreement with previously reported emission sources (Derudi et al., 2013; Morawska et al., 2013). Congeners with 2-3 aromatic rings (fluorene, phenanthrene, anthracene, and fluoranthene) showed a mixed trend: $\mathrm{I} / \mathrm{O}>1$ at S2, S6, S10, and $\mathrm{I} / \mathrm{O}<1$ at S7, S8, S9. The remaining PAHs (i.e. with 4-6 aromatic rings) exhibited I/O ratios lower than 1 at all schools (with exception to S7), indicating outdoor origin of these contaminants. Moreover, at S8 and S10, these ratios were even $<0.5$ suggesting that outdoor sources of 4-6 ringed PAHs were prevailing. S7 was the only school at which I/O of 5-6 ring PAHs were consistently $>1$. No specific source of PAHs that could justify this occurrence was identified, but this could be caused by the combined effects of position/distance of the classrooms from the main street and the low frequency of ventilation in the respective rooms.

\subsubsection{Diagnostic concentration ratios}

Analysis of diagnostic ratios of individual PAH congeners can provide further insight regarding PAHs origin. The ratios that were calculated for indoor PAHs at the ten primary schools are presented in Table 4. Ratio between total concentration of PAHs with 2 and 3-rings ( $\sum$ PAHs $s_{\text {LMW }}$ ) vs. the 4- to 6-ring ones ( $\Sigma$ PAHs HMw $_{\text {) }}$ differentiates between petrogenic and pyrogenic origin with values lower than unity indicating pyrogenic origin of PAHs (Krugly et al., 2014; Zhang et al., 2008). At S2, $\mathrm{S} 5, \mathrm{~S} 7, \mathrm{~S} 8$, and $\mathrm{S10}$, the values of this diagnostic ratio were $<1$, hence pointing towards a pyrogenic source of PAHs. Emissions from vehicular traffic were the most probable source of PAHs at these schools. S2, S8, and S10 were located next to roads and/or streets with a busy traffic (Table 1) whereas S7 was located in residential area; at these schools possible PAH sources included vehicular emissions and fuel burning. S5 was located in the residential area with traffic streets and potential impact of an industrial site in the vicinity. In agreement with these findings, values of $\mathrm{B}[a] \mathrm{A} / \mathrm{Chry}$ were higher than 0.35 at all schools and thus indicated vehicular emissions and/or fuel combustions (Krugly et al., 2014); similarly the values of InP/(InP + B[ghi]P) implied contributions of traffic emissions (specifically from diesel combustions; Table 4; Kavouras et al., 2001; Pio et al., 2001; Rogge et al., 1993a). It is necessary to point out that $\mathrm{B}[$ ghi $] \mathrm{P}$ is being often used as marker of diesel emissions but the levels obtained in this work were relatively low (6-8\% of $\Sigma$ PAHs). However, in the series of previous works that assessed ambient 


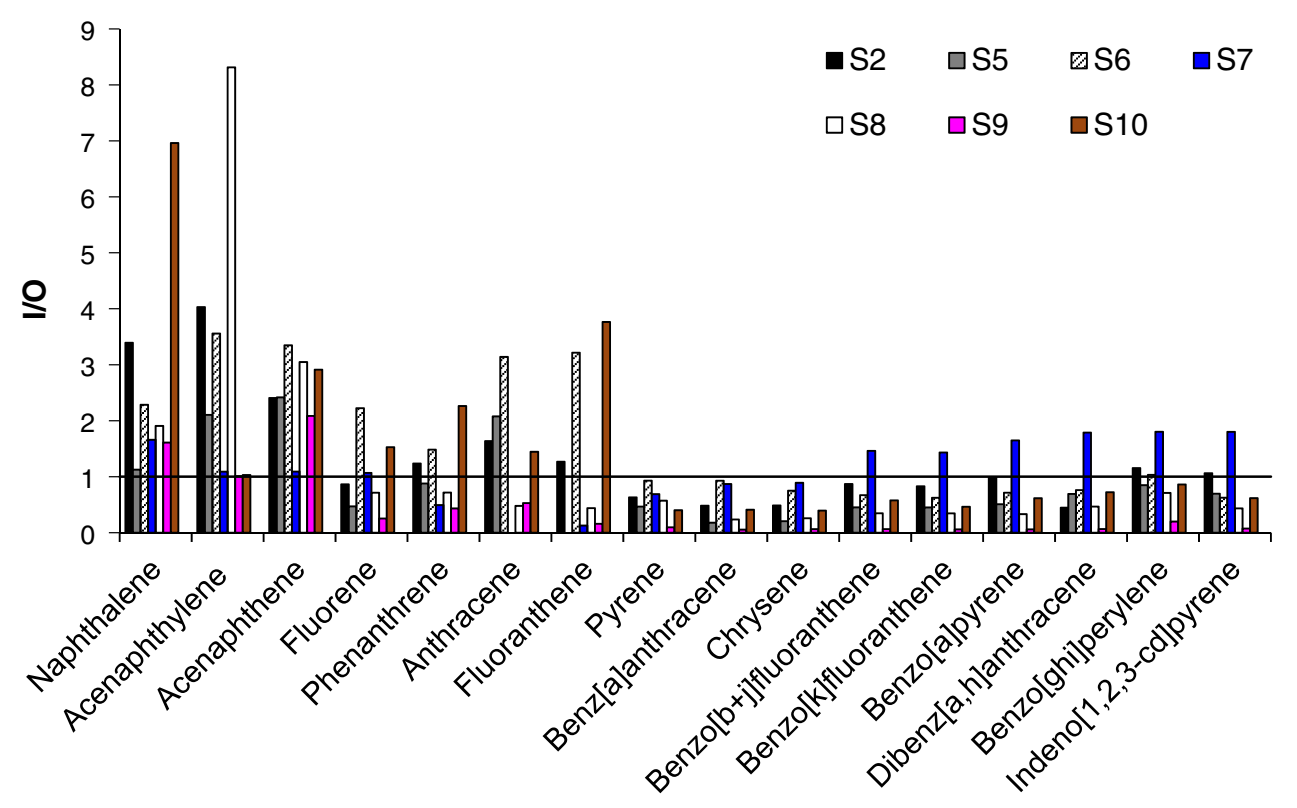

Fig. 2. Indoor to outdoor (I/O) $\mathrm{PM}_{2.5}$-bound $\mathrm{PAH}$ ratios at studied schools (S1-S10).

Table 4

Diagnostic ratios for indoor $\mathrm{PM}_{2.5}$-bound PAHs at the ten primary schools.

\begin{tabular}{|c|c|c|c|c|c|c|c|c|c|c|c|c|c|}
\hline \multirow[t]{2}{*}{ Ratio } & \multicolumn{10}{|c|}{ This study } & \multirow[t]{2}{*}{ Value } & \multirow[t]{2}{*}{ Source } & \multirow[t]{2}{*}{ Reference } \\
\hline & $\mathrm{S} 1$ & $\mathrm{~S} 2$ & S3 & S4 & S5 & S6 & S7 & S8 & S9 & S10 & & & \\
\hline${ }^{\mathrm{a}} \mathrm{ZPAHs} \mathrm{LMW}_{\mathrm{LM}} / \mathrm{ZPAH}_{\mathrm{SHMW}}$ & $\begin{array}{l}3.58 \\
\pm 0.56\end{array}$ & $\begin{array}{l}0.59 \\
\pm 0.18\end{array}$ & $\begin{array}{l}1.78 \\
\pm 0.16\end{array}$ & $\begin{array}{l}6.39 \\
\pm 0.57\end{array}$ & $\begin{array}{l}0.90 \\
\pm 0.05\end{array}$ & $\begin{array}{l}2.98 \\
\pm 0.59\end{array}$ & $\begin{array}{l}0.53 \\
\pm 0.14\end{array}$ & $\begin{array}{l}0.99 \\
\pm 0.12\end{array}$ & $\begin{array}{l}3.33 \pm \\
1.7\end{array}$ & $\begin{array}{l}0.66 \\
\pm 0.14\end{array}$ & $\begin{array}{l}>1 \\
<1\end{array}$ & $\begin{array}{l}\text { Petrogenic } \\
\text { Pyrogenic }\end{array}$ & Zhang et al. (2008) \\
\hline $\mathrm{B}[a] \mathrm{A} /$ Chry & $\begin{array}{l}0.36 \\
\pm 0.06\end{array}$ & $\begin{array}{l}0.50 \\
\pm 0.03\end{array}$ & $\begin{array}{l}0.52 \\
\pm 0.04\end{array}$ & $\begin{array}{l}0.37 \\
\pm 0.04\end{array}$ & $\begin{array}{l}0.45 \\
\pm 0.04\end{array}$ & $\begin{array}{l}0.44 \\
\pm 0.03\end{array}$ & $\begin{array}{l}0.42 \\
\pm 0.01\end{array}$ & $\begin{array}{l}0.39 \\
\pm 0.03\end{array}$ & $\begin{array}{l}0.36 \\
\pm \\
\pm 0.04\end{array}$ & $\begin{array}{l}0.46 \\
\pm 0.03\end{array}$ & $>0.35$ & $\begin{array}{l}\text { Fuel } \\
\text { combustion }\end{array}$ & Krugly et al. (2014) \\
\hline $\operatorname{InP} /(\operatorname{InP}+\mathrm{B}[$ ghi] $\mathrm{P})$ & $\begin{array}{l}0.42 \\
\pm 0.15\end{array}$ & $\begin{array}{l}0.50 \\
\pm 0.24\end{array}$ & $\begin{array}{l}0.46 \\
\pm 0.24\end{array}$ & $\begin{array}{l}0.33 \\
\pm 0.11\end{array}$ & $\begin{array}{l}0.47 \\
\pm 0.24\end{array}$ & $\begin{array}{l}0.36 \\
\pm 0.11\end{array}$ & $\begin{array}{l}0.50 \\
\pm 0.19\end{array}$ & $\begin{array}{l}0.43 \\
\pm 0.35\end{array}$ & $\begin{array}{l}0.27 \\
\pm 0.12\end{array}$ & $\begin{array}{l}0.41 \\
\pm 0.20\end{array}$ & $\begin{array}{l}0.21-0.22 \\
0.35-0.70 \\
0.56 \\
0.62 \\
0.36\end{array}$ & $\begin{array}{l}\text { Gasoline } \\
\text { cars } \\
\text { Diesel } \\
\text { emissions } \\
\text { Coal } \\
\text { combustion } \\
\text { Wood } \\
\text { combustion } \\
\text { Road dust }\end{array}$ & $\begin{array}{l}\text { Gogou et al. (1996), Grimmer et } \\
\text { al. (1983), Kavouras et al. } \\
\text { (2001), Pio et al. (2001), } \\
\text { Ravindra et al. (2008) and } \\
\text { Rogge et al. (1993a) }\end{array}$ \\
\hline $\mathrm{B}[b] \mathrm{F} / \mathrm{B}[k] \mathrm{F}$ & $\begin{array}{l}5.75 \\
\pm 0.20\end{array}$ & $\begin{array}{l}4.98 \\
\pm 0.13\end{array}$ & $\begin{array}{l}5.23 \\
\pm 0.10\end{array}$ & $\begin{array}{l}5.12 \\
\pm 0.23\end{array}$ & $\begin{array}{l}5.03 \\
\pm 0.25\end{array}$ & $\begin{array}{l}5.27 \\
\pm 0.28\end{array}$ & $\begin{array}{l}5.51 \\
\pm 0.34\end{array}$ & $\begin{array}{l}5.58 \\
\pm 0.22\end{array}$ & $\begin{array}{l}5.67 \\
\pm 0.14\end{array}$ & $\begin{array}{l}6.31 \pm \\
1.05\end{array}$ & $>0.5$ & Diesel & Ravindra et al. (2008) \\
\hline $\mathrm{B}[$ ghi $] \mathrm{P} / \mathrm{B}[a] \mathrm{P}$ & $\begin{array}{l}2.29 \\
\pm 0.17\end{array}$ & $\begin{array}{l}1.15 \\
\pm 0.07\end{array}$ & $\begin{array}{l}1.57 \\
\pm 0.08\end{array}$ & $\begin{array}{l}3.95 \\
\pm 0.12\end{array}$ & $\begin{array}{l}1.51 \\
\pm 0.07\end{array}$ & $\begin{array}{l}2.09 \\
\pm 0.31\end{array}$ & $\begin{array}{l}1.67 \\
\pm 0.30\end{array}$ & $\begin{array}{l}1.96 \\
\pm 0.27\end{array}$ & $\begin{array}{l}4.11 \pm \\
1.11\end{array}$ & $\begin{array}{l}2.16 \\
\pm 0.30\end{array}$ & $\begin{array}{l}1.2-2.2 \\
2.5-3.3 \\
0.86,0.91\end{array}$ & $\begin{array}{l}\text { Diesel cars } \\
\text { Gasoline } \\
\text { cars } \\
\text { Road dust }\end{array}$ & $\begin{array}{l}\text { Oda et al. (2001) and Rogge et } \\
\text { al. (1993a, 1993b) }\end{array}$ \\
\hline $\mathrm{B}[a] \mathrm{P} /(\mathrm{B}[a] \mathrm{P}+$ Chry $)$ & $\begin{array}{l}0.67 \\
\pm 0.06\end{array}$ & $\begin{array}{l}0.73 \\
\pm 0.03\end{array}$ & $\begin{array}{l}0.69 \\
\pm 0.03\end{array}$ & $\begin{array}{l}0.64 \\
\pm 0.01\end{array}$ & $\begin{array}{l}0.77 \\
\pm 0.04\end{array}$ & $\begin{array}{l}0.57 \\
\pm 0.06\end{array}$ & $\begin{array}{l}0.67 \\
\pm 0.03\end{array}$ & $\begin{array}{l}0.67 \\
\pm 0.04\end{array}$ & $\begin{array}{l}0.51 \\
\pm 0.01\end{array}$ & $\begin{array}{l}0.66 \\
\pm 0.05\end{array}$ & $\begin{array}{l}0.5 \\
0.73\end{array}$ & $\begin{array}{l}\text { Diesel } \\
\text { Gasoline }\end{array}$ & Ravindra et al. (2008) \\
\hline $\mathrm{B}[a] \mathrm{A} /(\mathrm{B}[a] \mathrm{A}+$ Chry $)$ & $\begin{array}{l}0.26 \\
\pm 0.03\end{array}$ & $\begin{array}{l}0.33 \\
\pm 0.01\end{array}$ & $\begin{array}{l}0.34 \\
\pm 0.02\end{array}$ & $\begin{array}{l}0.27 \\
\pm 0.02\end{array}$ & $\begin{array}{l}0.31 \\
\pm 0.02\end{array}$ & $\begin{array}{l}0.31 \\
\pm 0.01\end{array}$ & $\begin{array}{l}0.30 \\
\pm 0.01\end{array}$ & $\begin{array}{l}0.28 \\
\pm 0.03\end{array}$ & $\begin{array}{l}0.27 \\
\pm 0.02\end{array}$ & $\begin{array}{l}0.32 \\
\pm 0.03\end{array}$ & $\begin{array}{l}0.40 \\
0.38-0.64 \\
0.76 \\
0.43\end{array}$ & $\begin{array}{l}\text { Diesel } \\
\text { Gasoline } \\
\text { Wood } \\
\text { combustion }\end{array}$ & $\begin{array}{l}\text { Li and Kamens (1993), Manoli } \\
\text { et al. (2004) and Sicre et al. } \\
\text { (1987) }\end{array}$ \\
\hline $\mathrm{B}[b+k] \mathrm{F} / \mathrm{B}[g h i] \mathrm{P}$ & $\begin{array}{l}0.89 \\
\pm 0.07\end{array}$ & $\begin{array}{l}1.90 \\
\pm 0.15\end{array}$ & $\begin{array}{l}1.09 \\
\pm 0.19\end{array}$ & $\begin{array}{l}0.29 \\
\pm 0.05\end{array}$ & $\begin{array}{l}1.19 \\
\pm 025\end{array}$ & $\begin{array}{l}0.80 \\
\pm 0.07\end{array}$ & $\begin{array}{l}2.15 \\
\pm 0.07\end{array}$ & $\begin{array}{l}1.33 \\
\pm 0.23\end{array}$ & $\begin{array}{l}0.24 \\
\pm 0.22\end{array}$ & $\begin{array}{l}0.75 \\
\pm 0.11\end{array}$ & $\begin{array}{l}1.60 \\
0.33 \\
2.18\end{array}$ & $\begin{array}{l}\text { Diesel cars } \\
\text { Gasoline } \\
\text { cars } \\
\text { Wood } \\
\text { combustion }\end{array}$ & Li and Kamens (1993) \\
\hline Flu/(Flu + Pyr) & $\begin{array}{l}0.25 \\
\pm 0.04\end{array}$ & $\begin{array}{l}0.06 \\
\pm 0.01\end{array}$ & $\begin{array}{l}0.08 \\
\pm 0.01\end{array}$ & $\begin{array}{l}0.17 \\
\pm 0.02\end{array}$ & $\begin{array}{l}0.06 \\
\pm 0.01\end{array}$ & $\begin{array}{l}0.13 \\
\pm 0.03\end{array}$ & $\begin{array}{l}0.06 \\
\pm 0.02\end{array}$ & $\begin{array}{l}0.07 \\
\pm 0.01\end{array}$ & $\begin{array}{l}0.11 \\
\pm 0.08\end{array}$ & $\begin{array}{l}0.12 \\
\pm 0.01\end{array}$ & $\begin{array}{l}>0.5 \\
<0.5\end{array}$ & $\begin{array}{l}\text { Diesel } \\
\text { Gasoline }\end{array}$ & Ravindra et al. (2008) \\
\hline Phe/(Phe + Ant) & $\begin{array}{l}0.83 \\
\pm 0.01\end{array}$ & $\begin{array}{l}0.98 \\
\pm 0.02\end{array}$ & $\begin{array}{l}0.92 \\
\pm 0.06\end{array}$ & $\begin{array}{l}0.95 \\
\pm 0.01\end{array}$ & $\begin{array}{l}0.96 \\
\pm 0.02\end{array}$ & $\begin{array}{l}0.93 \\
\pm 0.01\end{array}$ & $\begin{array}{l}0.91 \\
\pm 0.04\end{array}$ & $\begin{array}{l}0.96 \\
\pm 0.02\end{array}$ & $\begin{array}{l}0.95 \\
\pm 0.03\end{array}$ & $\begin{array}{l}0.99 \\
\pm 0.01\end{array}$ & $>0.7$ & $\begin{array}{l}\text { Lubricant } \\
\text { oils and } \\
\text { fossil fuels }\end{array}$ & Mirante et al. (2013) \\
\hline
\end{tabular}

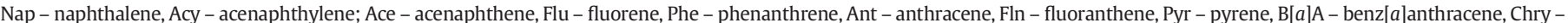

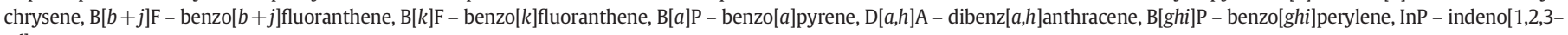
cd]pyrene.

a Total concentration of 2- and 3-rings PAHs ( 2 PAHs $s_{\mathrm{LMw}}$ ), and 4-6-rings PAHs ( $2 \mathrm{PAHs} \mathrm{HMw}_{\mathrm{HMw}}$ ). 
PAHs and their source identification for Oporto Metropolitan Area (Castro et al., 2009; Slezakova et al., 2010, 2011b, 2013b), this congener has not been the predominant compound (though vehicular emissions were identified as the major emission source). The results instead demonstrated higher abundances of other traffic emission indicators, such as dibenz $[a, h]$ anthracene and/or benzo $[b+j]$ fluoranthene (Callén et al., 2013; Ravindra et al., 2008), similarly to results of the this study.

At S1, S3, S4, S6 and S9 the diagnostic ratios of $\Sigma \mathrm{PAHs}_{\mathrm{LMW}}$ to $\Sigma \mathrm{PAHs}_{\mathrm{HMW}}$ were $>1$, suggesting petrogenic origin. These unexpected findings could be caused by emissions from oil refinery (combined with a petrochemical complex) that is located approximately $15 \mathrm{~km}$ west from the respective area. It also should be remarked that diagnostic ratios are merely rudimentary indicators of PAH origin (Galarneau, 2008) and need to be interpreted carefully. Numerous studies have demonstrated their limitations as their values can be altered to a different extent due to environmental degradation and phase transfers (Dvorská et al., 2011; Galarneau, 2008; Katsoyiannis et al., 2007, 2011; Tobiszewski and Namieśnik, 2012; Usenko et al., 2010). In addition, atmospheric reactions between PAHs and other pollutants such as $\mathrm{NO}_{\mathrm{X}}$ and $\mathrm{O}_{3}$ can have further impact on the ratios values; Ravindra et al., 2008).

The rest of diagnostic ratios (Table 4) used for indoor PAHs at ten primary schools also pointed towards vehicular influences. The values of $\mathrm{B}[b] \mathrm{F} / \mathrm{B}[k] \mathrm{F}>0.5$ (Ravindra et al., 2008) indicated an influence of diesel emissions as well as the values of $\mathrm{B}[\mathrm{ghi}] \mathrm{P} / \mathrm{B}[a] \mathrm{P}$ which mostly ranged from 1.2-2.2. These results are further supported as diesel is the fuel mostly used for road transport accounting for $78.3 \%$ of the Portuguese consumption of vehicle fuels (INE, 2014). However, other diagnostic ratios that were applied in order to differentiate between types of vehicle emissions (i.e. diesel versus gasoline) and the obtained findings were not completely consistent. Values of $\mathrm{B}[a] \mathrm{P} /(\mathrm{B}[a] \mathrm{P}+$ Chry $), \mathrm{B}[a] \mathrm{A} /$ $(\mathrm{B}[a] \mathrm{A}+$ Chry), and $\mathrm{B}[b+k] \mathrm{F} / \mathrm{B}[$ ghi $] \mathrm{P}$ (Table 4$)$ indicated a mixed contribution of both types of emissions at the characterized schools. Values of Flu/(Flu + Pyr) can be used to distinguish between the exhausts origin with values $>0.5$ pointing towards diesel emissions while ratio $<0.5$ indicates gasoline type (Ravindra et al., 2008). The values of this ratio were very low (Table 4), therefore potentially indicating influence of gasoline emissions at schools (Li and Kamens, 1993). Lastly, at all ten schools, Phe/(Phe + Ant $)>0.7$ indicated emissions from lubricant oils and/or fossil fuels (Mirante et al., 2013). Based on the applied ratios analysis, it is possible to assume that vehicular traffic was a significant emission source for PAHs present in indoor air, with a mixed contribution from both diesel and gasoline fueled cars. In that regard it is necessary to emphasize the findings of a recent study by Finardi et al. (2015) which highlighted rather old technical literature concerning PAH emission signatures that lacks investigations on molecular signatures of new generation vehicles, biomass burning technologies, boilers and waste treatment plants, so that the available reference values of currently used diagnostic ratios sometimes seem unusable to assign unequivocally PAHs to specific sources.

Finally, seasonal variations of atmospheric PAHs have been previously demonstrated. Using different diagnostic ratios of PAHs, Jedynska et al. (2014) identified traffic emissions (diesel and gasoline fueled vehicles) as the main source of PAHs, and wood burning as the second important contribution causing a higher impact at regional background stations. Finardi et al. (2015) analysed several diagnostic ratios and rates of their seasonal variability, allowing identification of different sources with a prevalent impact of residential combustion during winter and of traffic emissions in summer. However, the analysis of diagnostic ratios applied to indoor levels of PAHs during winter and spring season (Table 5S) did not reveal a prevailing contribution of different emission sources for the two seasons. Nevertheless, in a view of the risk assessment, this study was designed to monitor PAHs during one complete trimester of a school year, which resulted only in $1 / 3$ of the data collected for the spring season. Thus, further evaluation focusing longer sampling of PAHs (both in ambient air and indoor area) would be required in order to characterize in depth seasonal variation of ambient and indoor PAHs in Oporto metropolitan Area.

\subsubsection{Risk analysis}

The TEF-adjusted levels for 18 PAHs (indoors and outdoors) of studied schools are shown in Tables $6 \mathrm{~S}$ and $7 \mathrm{~S}$, respectively. Dibenz $[a, h]$ anthracene exhibited the second highest levels (approximately between 5 and $25 \%$ to $\Sigma_{\text {PAHs }}$ in indoor air), and was the most important PAH of $\Sigma_{\text {TEF-PAHs }}$, most likely due its high value of TEF. On average dibenz $[a, h]$ anthracene contributed $93 \%$ of both indoor (range 84-95\%) and outdoor (92-94\%) $\Sigma_{\text {TEF-PAHs }}$ at the selected schools. Benzo[a]pyrene (that is among 18 studied PAHs the only ascertained carcinogen to human health) was the second most predominant $\mathrm{PAH}$ of $\Sigma_{\text {TEF-PAHs }}$ accounting for 3-11\% at indoor and $4-6 \%$ at outdoor air.

Lung cancer risk due to total school exposure (i.e. sum of both indoors and outdoors) was calculated according to WHO (benzo[a]pyrene unit risk value $8.7 \times 10^{-5}$ per $\mathrm{ng} \mathrm{m}^{-3}$, considering exposure of 70 years; WHO, 2010); values ranged between $6.5 \times 10^{-5}$ (at S9) and $8.7 \times 10^{-4}$ (S2). Thus lung cancer risk estimated for the characterized primary schools surpassed WHO recommended threshold of $10^{-5}$ (Boström et al., 2002), being about 7-87 times higher than the recommended guideline. The respective values of risk though might be even higher as they were estimated considering $6 \mathrm{~h}$ of indoor exposure time (as spent in classrooms). Nevertheless, in the remaining school time (additional 1$1.5 \mathrm{~h}$ ) children move between other school indoor microenvironments (libraries, gymnasiums, canteens, schools public halls) and the exposures in these places could add to overall indoor exposure to PAHs (and consequently increase the respective risk). Therefore, future assessment of PAH levels and risk in these school microenvironments would be of a great importance.

The target carcinogenic risk calculated for students of primary schools (8-10 years old) and school personnel were assessed using USEPA methodology and the means are presented in Table 8S. USEPA recommends a $10^{-6}$ threshold (USEPA, 1989), nonetheless the accumulative carcinogenic risks should not be higher than the more restrictive level of $10^{-4}$. Target risk due to indoor exposure $\left(\Sigma \mathrm{TR}_{\text {Indoor }}\right)$ exceeded the recommended guideline at all schools with exception to S4 and S9 (due to much lower levels of the respective PAH indoors; Table 2), whereas risk due to outdoor exposure ( $\Sigma \mathrm{TR}_{\text {Outdoor }}$ ) was below $10^{-6}$ and thus negligible at all schools. Specifically, $\Sigma T_{\text {Indoor }}$ were 2-10 times higher than $\Sigma T R_{\text {Outdoor }}$ probably due to prolonged periods that children spent indoors ( $6 \mathrm{~h}$ indoors in classrooms versus $1-1.25 \mathrm{~h}$ outdoors). Overall, $\Sigma \mathrm{TR}_{\text {Indoor }}$ contributed $70-90 \%$ of total school risk ( $\Sigma \mathrm{TR}_{\mathrm{School}}$, i.e. sum of both indoors and outdoors). Considering these high contributions of indoor risk, future studies should be conducted allowing a deeper understanding of child exposure and the health consequences in schools in order to develop and implement the respective guidelines which are essential for these environments.

The carcinogenic risk due to total (indoor and outdoor) school exposure was also calculated. Results in Fig. 3a show that with exceptions to S4 (absence of outdoor PAH assessment) and S9 (significantly lower, i.e. the lowest, indoor PAHs; Table 2), carcinogenic risk exceeded (up to 22 times) the recommended guideline at all schools. For adults, carcinogenic risk due to total exposure exceeded (2-166 times) the recommended level of $10^{-6}$ at all schools (Fig. 3b), at S2 being even higher than the more stringent guideline of $10^{-4}$. For adult populations of school personnel, the risk of overall school exposure ( $\left.2 T_{S c h o o l}\right)$ were about 8 times greater than children, probably due to the longer exposure duration (15 versus 2 years).

In order to decrease the overall risk of adverse health outcomes, improved management of (indoor) air pollution is necessary. Regarding PAHs, this implies reducing personal exposure to various indoor combustion sources (heaters, candles, essences and stick burning, open fire cooking) but also reduced exposure in indoor microenvironments near sources such as traffic, use of protective equipment (for polluted occupational settings), and various interventions to modify individual 


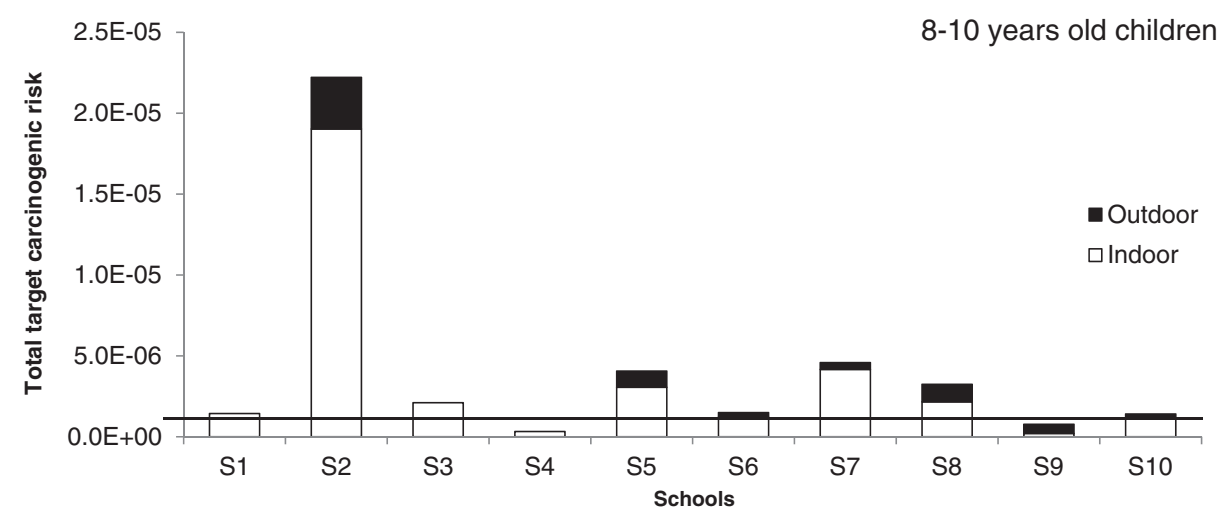

(a)

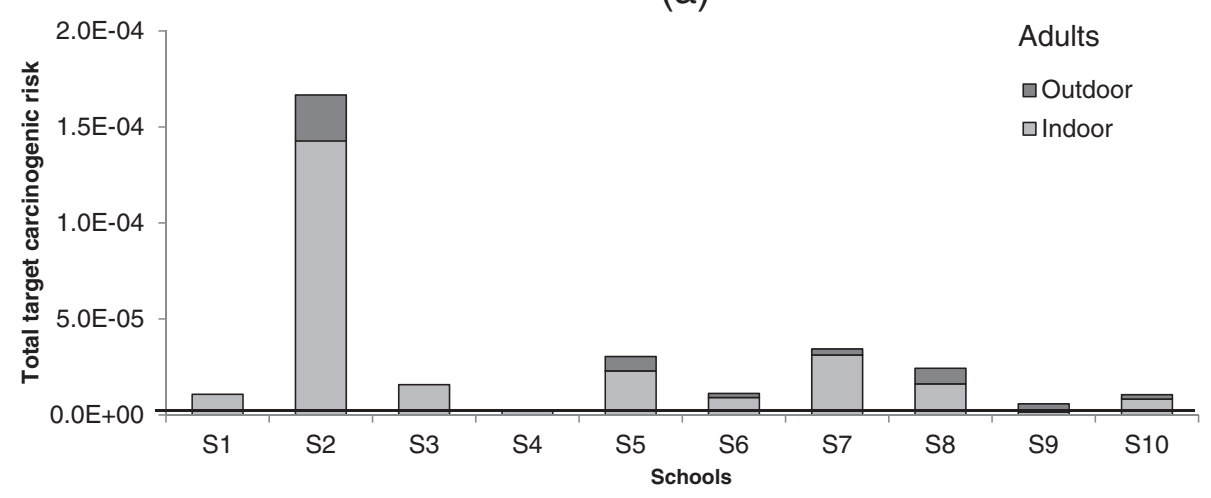

(b)

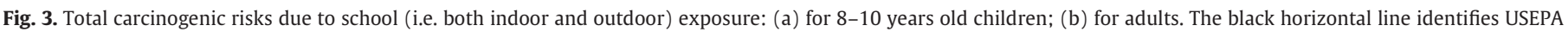
recommended guideline level of $10^{-6}$.

susceptibility. It is understandable that contribution of indoor pollutants and of those generated outdoors to personal exposures depends on multiple factors (such as building characteristics, presence of indoor emission sources and personal activities, type of pollutants, and etc.). These parameters need to be taken into the account (on individual levels) when assessing, and reducing, the personal exposure to these harmful pollutants (Laumbach et al., 2015).

\section{Conclusions}

This work provides information concerning $\mathrm{PM}_{2.5}$-bound PAHs levels and risk in primary schools environments as there is few data in literature on this topic. Urban school situated in the most polluted site (S2) exhibited the highest levels of indoor PAHs, whereas the lowest indoor concentrations were found for a school situated in residential area (S9). I/O ratios of congeners with $2-3$ aromatic rings demonstrated the influence of indoor emission sources whereas the occurrence of higher molecular weight PAH congeners (4-6 aromatic rings) was mainly caused by infiltration of ambient emissions indoors. Consequently, diagnostic ratios demonstrated emissions from vehicular fuel combustions (both of diesel and gasoline origin) as the main source of indoor particulate-bound PAHs.

Total carcinogenic risk due to indoor school exposure was 2-10 times higher than for outdoors, mainly due to the extended durations of time that primary students are indoors. The risk assessment revealed that cancer risk of 8-10 years old school children and adults exceeded (up to 22 times, and 2-166 times for children and schools staff, respectively) the recommended USEPA guideline; WHO health-based level of $10^{-5}$ was exceeded 7-87 times, which may potentially cause negative health outcomes of the exposed populations. It is necessary to point out that the risk assessment was conducted using PAH levels averaged over the period of one school trimester (i.e. 10 weeks); however, PAH levels in air are season-dependent with winter concentrations being typically higher than the summer ones (Finardi et al., 2015) which reflect on the estimated risk values and over findings of this study. Finally, the sampling approach (i.e. duration, period, choice of sampling method and technology) could cause some additional bias (Lewis et al., 2016). Thus future study combining the traditional and novel measures for personal exposures (Koehler and Peters, 2015) may provide alternate strategies for exposure assessment and yield stronger, more precise findings on associations with adverse health outcomes.

\section{Ethical statement}

Human subjects were not directly involved in this study. Full information about the measurements conducted in this work was provided to all students and teaching staff using the respective spaces as well as to the parents and boards of directors of all primary schools.

\section{Acknowledgments}

This work was supported by European Union (FEDER funds through COMPETE) and National Funds (Fundação para a Ciência e Tecnologia) through projects UID/QUI/50006/2013 and POCI-01-0145-FEDER006939, by the FCT/MEC with national funds and co-funded by FEDER in the scope of the P2020 Partnership Agreement. Additional financial support was provided by Fundação para Ciência e Tecnologia through project PTDC/DTP-SAP/1522/2012 (ARIA: How indoor air quality can affect children allergies and asthma) and fellowships SFRH/BPD//105100/ 2014 (Klara Slezakova) and SFRH/BD/80113/2011 (Marta Oliveira). 


\section{Appendix A. Supplementary data}

Supplementary data to this article can be found online at http://dx. doi.org/10.1016/j.scitotenv.2016.09.195.

\section{References}

Alves, C.A., Urban, R.C., Pegas, P.N., Nunes, T., 2014. Indoor/outdoor relationships between PM10 and associated organic compounds in a primary school. Aerosol Air Qual. Res. $14,86-98$.

Amato, F., Rivas, I., Viana, M., Moreno, T., Bouso, L., Reche, C., Àlvarez-Pedrerol, M., Alastuey, A., Sunyer, J., Querol, X., 2014. Sources of indoor and outdoor $\mathrm{PM}_{2.5}$ concentrations in primary schools. Sci. Total Environ. 490, 757-765.

Annesi-Maesano, I., Moreau, D., Caillaud, D., Lavaud, F., Le Moullec, Y., Taytard, A., Pauli, G. Charpin, D., 2007. Residential proximity fine particles related to allergic sensitisation and asthma in primary school children. Respir. Med. 101, 1721-1729.

Bae, S., Pan, X.C., Kim, S.Y., Park, K., Kim, Y.H., Kim, H., Hong, Y.C., 2010. Exposures to particulate matter and polycyclic aromatic hydrocarbons and oxidative stress in schoolchildren. Environ. Health Perspect. 118, 579-583.

Boström, C.E., Gerde, P., Hanberg, A., Jernstrom, B., Johansson, C., Kyrklund, T., Rannug, A. Tornqvist, M., Victorin, K., Westerholm, R., 2002. Cancer risk assessments, indicators, and guidelines for polycyclic aromatic hydrocarbons in the ambient air. Environ. Health Perspect. 10, 451-488.

Burtscher, H., Schüepp, K., 2012. The occurrence of ultrafine particles in the specific environment of children. Paediatr. Respir. Rev. 13, 89-94.

Callén, M.S., López, J.M., Iturmendi, A., Mastral, A.M., 2013. Nature and sources of particle associated polycyclic aromatic hydrocarbons $(\mathrm{PAH})$ in the atmospheric environment of an urban area. Environ. Pollut. 183, 166-174.

Carpente, D.O., Bushkin-Bedient, S., 2013. Exposure to chemicals and radiation during childhood and risk for cancer later in life. J. Adolesc. Health 52 (5 Suppl), S21-S29.

Castro, D., Slezakova, K., Delerue-Matos, C., Alvim-Ferraz, M.C., Morais, S., Pereira, M.C. 2011. Polycyclic aromatic hydrocarbons in gas and particulate phases of indoor environment influenced by tobacco smoke: levels, phase distribution and health risks. Atmos. Environ. 45, 1799-1808.

Castro, D., Slezakova, K., Oliva-Teles, M.T., Delerue-Matos, C., Alvim-Ferraz, M.C., Morais, S., Pereira, M.C., 2009. Analysis of polycyclic aromatic hydrocarbons in atmospheric particulate samples by microwave-assisted extraction and liquid chromatography. J. Sep. Sci. 32, 501-510

Chen, C., Zhao, B., Zhou, W.T., Jiang, X.Y., Tan, Z.C., 2012. A methodology for predicting particle penetration factor through cracks of windows and doors for actual engineering application. Build. Environ. 47, 339-348.

Cirillo, T., Montuori, P., Mainardi, P., Russo, I., Triassi, M., Amodio-Cocchieri, R., 2006 Multipathway polycyclic aromatic hydrocarbon and pyrene exposure among children living in Campania (Italy). J. Environ. Sci. Health A. Tox. Hazard Subst. Environ. Eng. 41, 2089-2107.

Derudi, M., Gelosa, S., Sliepcevich, A., Cattaneo, A., Cavallo, D., Rota, R., Nano, G., 2013. Emission of air pollutants from burning candles with different composition in indoor environments. Environ. Sci. Pollut. Res. 21, 4320-4330.

Directive 2004/107/EC, 2005. Directive of the European Parliament and of the council relating to arsenic, cadmium, mercury, nickel and polycyclic aromatic hydrocarbons in ambient air. Off. J. Eur. Union L23, 3-16.

Dvorská, A., Lammel, G., Klánová, J., 2011. Use of diagnostic ratios for studying source apportionment and reactivity of ambient polycyclic aromatic hydrocarbons over Central Europe. Atmos. Environ. 45, 420-427.

Eiguren-Fernandez, A., Avol, E.L., Thurairatnam, S., Hakami, M., Froines, J.R., Miguel, A.H. 2007. Seasonal influence on vapor-and particle-phase polycyclic aromatic hydrocarbon concentrations in school communities located in southern California. Aerosol Sci. Technol. 41, 438-446.

Finardi, S., Radice, P., Cecinato, A., Gariazzo, C., Gherardi, M., Romagnoli, P., 2015. Seasonal variation of PAHs concentration and source attribution through diagnostic ratios analysis. Urban Climate http://dx.doi.org/10.1016/j.uclim.2015.12.001 in press.

Foos, B., Marty, M., Schwartz, J., Bennett, W., Moya, J., Jarabek, A.M., Salmon, A.G., 2008. Focusing on children's inhalation dosimetry and health effects for risk assessment: an introduction. J. Toxicol. Environ. Health - Part A: Current Issues 71, 149-165.

Galarneau, E., 2008. Source specificity and atmospheric processing of airborne PAHs: implications for source apportionment. Atmos. Environ. 42, 8139-8149.

Gatto, M.P., Gariazzo, C., Gordiani, A., L'Episcopo, N., Gherardi, M., 2013. Children and elders exposure assessment to particle-bound polycyclic aromatic hydrocarbons (PAHs) in the city of Rome Italy. Environ. Sci. Pollut. Res. 21, 13152-13159.

Gilliland, F.D., 2009. Outdoor air pollution, genetic susceptibility, and asthma management: opportunities for intervention to reduce the burden of asthma. Pediatrics 123 (Suppl. 3), S168-S173.

Gogou, A., Stratigakis, N., Kanakidou, M., Stefanou, E.G., 1996. Organic aerosols in Eastern Mediterranean: components source reconciliation by using molecular markers and atmospheric back trajectories. Org. Geochem. 25, 79-96.

Grimmer, G., Jacob, J., Naujack, K.W., Dettbarn, G., 1983. Determination of polycyclic aromatic compounds emitted from brown-coal-fired residential stoves by gas chromatography/gas spectrometry. Anal. Chem. 55, 892-900.

Hanedar, A., Alp, K., Kaynak, B., Avşar, E., 2014. Toxicity evaluation and source apportionment of polycyclic aromatic hydrocarbons (PAHs) at three stations in Istanbul, Turkey. Sci. Total Environ. 488-489, 437-446.

Haritash, A.K., Kaushik, C.P., 2009. Biodegradation aspects of polycyclic aromatic hydrocarbons (PAHs): a review. J. Hazard. Mater. 169 (1-3), 1-15
Hornung, R.W., Reed, L.D., 1990. Estimation of average concentration in the presence of nondetectable values. Appl. Occup. Environ. Hyg. 5, 46-51.

IARC Working Group on the Evaluation of Carcinogenic Risks to Humans, 2002s. Some traditional herbal medicines, some mycotoxins, naphthalene and styrene. IARC Monogr. Eval. Carcinog. Risks Hum. 82, 1-556.

IARC Working Group on the Evaluation of Carcinogenic Risks to Humans (IARC), 2010s. Some non-heterocyclic polycyclic aromatic hydrocarbons and some related exposures. IARC Monogr. Eval. Carcinog. Risks Hum. 92, 1-853.

Instituto Nacional de Estatística (INE), 2014. Statistical Yearbook of Portugal 2013. Instituto Nacional de Estatística, I.P, Lisbon, Portugal.

Jedynska, A., Hoek, G., Eeftens, M., Cyrysc, J., Keuken, M., Ampe, C., Beelen, R., Cesaroni, G., Forastiere, F., Cirach, M., de Hoogh, K., De Nazelleg, A., Madsen, C., Declercq, C., Eriksen, K.T., Katsouyanni, K., Makarem, A.H., Lanki, T., Meliefste, K. Nieuwenhuijsen, M., Oldenwening, M., Pennanen, A., Raaschou-Nielsen, O., Brunekreef, B., Kooter, I.M., 2014. Spatial variations of PAH, hopanes/steranes and $\mathrm{EC} / \mathrm{OC}$ concentrations within and between European study areas. Atmos. Environ. 87, 239-248.

Jovanović, M., Vučićević, B., Turanjanin, V., Živković, M., Spasojević, V., 2014. Investigation of indoor and outdoor air quality of the classrooms at a school in Serbia. Energy 77 , $42-48$.

Jyethi, D.S., Khillare, P.S., Sarkar, S., 2014. Risk assessment of inhalation exposure to polycyclic aromatic hydrocarbons in school children. Environ. Sci. Pollut. Res. 21, 366-378.

Katsoyiannis, A., Sweetman, A.J., Jones, K.C., 2011. PAH molecular diagnostic ratios applied to atmospheric sources: a critical evaluation using two decades of source inventory and air concentration data from the UK. Environ. Sci. Technol. 45, 8897-8906.

Katsoyiannis, A., Terzi, E., Cai, Q.-Y., 2007. On the use of PAH molecular diagnostic ratios in sewage sludge for the understanding of the $\mathrm{PAH}$ sources. Is this use appropriate? Chemosphere 69, 1337-1339.

Kavouras, I.G., Koutrakis, P., Tsapakis, M., Lagoudaki, E., Stephanou, E.G., Von Baer, D. Oyola, P., 2001. Source apportionment of urban particulate aliphatic and polynuclea aromatic hydrocarbons (PAHs) using multivariate methods. Environ. Sci. Technol. 35, 2288-2294.

Kim, K.H., Pandey, S.K., Kabir, E., Susaya, J., Brown, R.J.C., 2011. The modern paradox of unregulated cooking activities and indoor air quality. J. Hazard. Mater. 195, 1-10.

Koehler, K.A., Peters, A., 2015. New methods for personal exposure monitoring for airborne particles. Curr. Environ. Health Rep. 2, 399-411.

Krugly, E., Martuzevicius, D., Sidaraviciute, R., Ciuzas, D., Prasauskas, T., Kauneliene, V., Stasiulaitiene, I., Kliucininkas, L., 2014. Characterization of particulate and vapor phase polycyclic aromatic hydrocarbons in indoor and outdoor air of primary schools. Atmos. Environ. 82, 298-306.

Laumbach, R., Meng, O., Kipen, H., 2015. What can individuals do to reduce personal health risks from air pollution? J. Thorac. Dis. 7, 96-107.

Lewis, A.C., Lee, J.D., Edwards, P.M., Shaw, M.D., Evans, M.J., Moller, S.J., Smith, K.R., Buckley, J.W., Ellis, M., Gillot, S.R., White, A., 2016. Evaluating the performance of low cost chemical sensors for air pollution research. Faraday Discuss. 189, 85-103.

Li, C.K., Kamens, R.M., 1993. The use of polycyclic aromatic hydrocarbons as source signatures in receptor modelling. Atmos. Environ. 27, 523-532.

Ma, W.L., Sun, D.Z., Shen, W.G., Yang, M., Qi, H., Liu, L.Y., Shen, J.M., Li, Y.F., 2011. Atmospheric concentrations, sources and gas-particle partitioning of PAHs in Beijing after the 29th Olympic Games. Environ. Pollut. 159 (7), 1794-18001.

Manoli, E., Kouras, A., Samara, C., 2004. Profile analysis of ambient and source emitted particle-bound polycyclic aromatic hydrocarbons from three sites in northern Greece. Chemosphere 56, 867-878.

Masih, J., Masih, A., Kulshrestha, A., Singhvi, R., Taneja, A., 2010. Characteristics of polycyclic aromatic hydrocarbons in indoor and outdoor atmosphere in the north central part of India. J. Hazard. Mater. 177, 190-198.

Merlo, D.F., Ceppi, M., Stagi, E., Bocchini, V., Sram, R.J., Rössner, P., 2007. 7. Toxicol. Lett $172(1-2), 60-67$.

Mirante, F., Alves, C., Pio, C., Pindado, O., Perez, R., Revuelta, M.A., Artiñano, B., 2013. Organic composition of size segregated atmospheric particulate matter, during summe and winter sampling campaigns at representative sites in Madrid, Spain. Atmos. Res. $132-133,345-361$

Morawska, L. Afshari, A, Bae, G.N., Buonanno, G., Chao, C.Y.H., Hänninen, O., Hofmann, O Isaxon, C., Jayaratne, E.R., Pasanen, P., Salthammer, P., Waring, M., Wierzbicka, A., 2013. Indoor aerosols: from personal exposure to risk assessment. Indoor Air 23, 462-487.

Moreno, T., Rivas, I., Bouso, L., Viana, M., Jones, T., Àlvarez-Pedrerol, M., Andrés Alastuey, A., Sunyer, J., Querol, X., 2014. Variations in school playground and classroom atmospheric particulate chemistry. Atmos. Environ. 91, 162-171.

Moshammer, H., Neuberger, M., 2003. The active surface of suspended particles as a predictor of lung function and pulmonary symptoms in Austrian school children. Atmos. Environ. 37, 1737-1744.

Neri, M., Bonassi, S., Knudsen, L.E., Sram, R.J., Holland, N., Ugolini, D., Merlo, D.F., 2006. Children's exposure to environmental pollutants and biomarkers of genetic damage. I. Overview and critical issues. Mutat. Res. Rev. Mutat Res. 612 (1), 1-13.

Oda, J., Nomura, S., Yasuhara, A., Shibamoto, T., 2001. Mobile sources of atmospheric polycyclic aromatic hydrocarbons in a roadway tunnel. Atmos. Environ. 35, 4819-4827.

Oliveira, M., Slezakova, K., Delerue-Matos, C. Pereira, M.C., Morais, S., 2015. Exposure to polycyclic aromatic hydrocarbons and assessment of potential risks in preschool children. Environ. Sci. Pollut. Res. 22 (18), 13892-13902.

Oliveira, M., Slezakova, K., Delerue-Matos, C., Pereira, M.C., Morais, S., 2016. Assessment of polycyclic aromatic hydrocarbons in indoor and outdoor air of preschool environments (3-5 years old children). Environ. Pollut. 208, 382-394.

Pereira, M.C., Santos, R.C., Alvim-Ferraz, M.C.M., 2007. Air quality improvements using European environment policies: a case study of $\mathrm{SO}_{2}$ in a coastal region in Portugal. J. Toxicol. Environ. Health - Part A: Current Issues 70, 347-351. 
Pio, C.A., Alves, C.A., Duarte, A.C., 2001. Identification, abundance and origin of atmospheric organic particulate matter in a Portuguese rural area. Atmos. Environ. 35 $1365-1375$

Pohl, H.R., Abadin, H.G., 2008. Chemical mixtures: evaluation of risk for child specific exposures in a multi-stressor environment. Toxicol. Appl. Pharmacol. 233, 116-125.

Qi, H., Li, W.L., Zhu, N.Z., Ma, W.L., Liu, L.Y., Zhang, F., Li, Y.F., 2014. Concentrations and sources of polycyclic aromatic hydrocarbons in indoor dust in China. Sci. Total Environ. 491-492, 100-107.

Ravindra, K., Sokhi, R., Grieken, R.V., 2008. Atmospheric polycyclic aromatic hydrocarbons: source attribution, emission factors and regulation. Atmos. Environ. 42 2895-2921.

Rivas, I., Viana, M., Moreno, T., Bouso, L., Pandolfi, M., Àlvarez-Pedrerol, M., Forns, J., Alastuey, A., Sunyer, J., Querol, X., 2015. Outdoor infiltration and indoor contribution of UFP and BC, OC, secondary inorganic ions and metals in $\mathrm{PM}_{2.5}$ in schools. Atmos. Environ. 106, 129-138.

Rivas, I., Viana, M., Moreno, T., Pandolfi, M., Amato, F., Reche, C., Bouso, L., ÀlvarezPedrerol, M., Alastuey, A., Sunyer, J., Querol, X., 2014. Child exposure to indoor and outdoor air pollutants in schools in Barcelona, Spain. Environ. Int. 69, 200-212.

Rogge, W.F., Hildemann, L.M., Mazurek, M.A., Cass, G.R., Simoneit, B.R.T., 1993a. Sources of fine organic aerosol. 2. Noncatalyst and catalyst-equipped automobiles and heavyduty diesel trucks. Environ. Sci. Technol. 27, 636-651.

Rogge, W.F., Hildemann, L.M., Mazurek, M.A., Cass, G.R., Simoneit, B.R.T, 1993b. Sources of fine organic aerosol. 3. Road dust, tire debris and organometallic brake lining dust: roads as sources and sinks. Environ. Sci. Technol. 27, 1892-1904.

Romagnoli, P., Balducci, C., Perilli, M., Gherardi, M., Gordiani, A., Gariazzo, C., Gatto, M.P. Cecinato, A., 2014. Indoor PAHs at schools, homes and offices in Rome, Italy. Atmos. Environ. 92, 51-59.

Ruchirawat, M., Navasumrit, P., Settachan, D., Autrup, H., 2006. Environmental impacts on children's health in Southeast Asia: genotoxic compounds in urban air. Ann. N. Y. Acad. Sci. 1076, 678-690.

Ruchirawat, M., Settachan, D., Navasumrit, P., Tuntawiroon, J., Autrup, H., 2007. Assessment of potential cancer risk in children exposed to urban air pollution in Bangkok, Thailand. Toxicol. Lett. 168, 200-209.

Samburova, V., Connolly, J., Gyawali, M., Yatavelli, R.L.N., Watts, A.C., Chakrabarty, R.K., Zielinska, B., Moosmüller, H., Khlystov, A., 2016. Polycyclic aromatic hydrocarbon in biomass-burning emissions and their contribution to light absorption and aeroso toxicity. Sci. Total Environ. 568, 391-401.

Sarigiannis, D.A., Karakitsios, S.P., Zikopoulos, D., Nikolaki, S., Kermenidou, M., 2015. Lung cancer risk from PAHs emitted from biomass combustion. Environ. Res. 137, 147-156.

Shen, G., Wei, S., Zhang, Y., Wang, R., Wang, B., Li, W., Shen, H., Huang, Y., Chen, Y., Chen, H., Wei, W., Tao, S., 2012. Emission of oxygenated polycyclic aromatic hydrocarbons from biomass pellet burning in a modern burner for cooking in China. Atmos. Environ. 60, 234-237.

Sicre, M.A., Marty, J.C., Saliot, A., Aparicio, X., Grimalt, J., Albaiges, S., 1987. Aliphatic and aromatic hydrocarbons in different sized aerosols over the Mediterranean Sea: occurrence and origin. Atmos. Environ. 21, 2247-2259.

Singh, D.K., Gupta, T., 2016. Effect through inhalation on human health of PM1 bound polycyclic aromatic hydrocarbons collected from foggy days in northern part of India. J. Hazard. Mater. 306, 257-268.

Slezakova, K., Castro, D., Begonha, A., Delerue-Matos, C., Alvim-Ferraz, M.C., Morais, S Pereira, M.C., 2011b. Air pollution from traffic emissions in Oporto, Portugal: health and environmental implications. Microchem. J. 99, 51-59.

Slezakova, K., Castro, D., Delerue-Matos, C., Alvim-Ferraz, M.C.M., Morais, S., Pereira, M.C. 2013a. Impact of vehicular traffic emissions on particulate-bound PAHs: levels and associated health risks. Atmos. Res. 127, 141-147.

Slezakova, K., Castro, D. Delerue-Matos, C., Morais, S, Pereira, M.C, 2014. Levels and risks of particulate-bound PAHs in indoor air influenced by tobacco smoke: a field measurement. Environ. Sci. Pollut. Res. 21, 4492-4501.

Slezakova, K., Castro, D., Pereira, M.C., Morais, S., Delerue-Matos, C., Alvim-Ferraz, M.C. 2010. Influence of traffic emissions on the carcinogenic polycyclic aromatic hydrocarbons in outdoor breathable particles. J. Air Waste Manage. Assoc. 60, 393-401.
Slezakova, K., Pires, J.C.M., Castro, D., Alvim-Ferraz, M.C.M., Delerue-Matos, C., Morais, S., Pereira, M.C., 2013b. PAH air pollution at a Portuguese urban area: carcinogenic risks and sources identification. Environ. Sci. Pollut. Res. 20 (6), 3932-3945.

Slezakova, K., Pires, J.C.M., Martins, F.G., Pereira, M.C., Alvim-Ferraz, M.C., 2011a. Identification of tobacco smoke components in indoor breathable particles by SEM-EDS. Atmos. Environ. 45, 863-872.

Tobiszewski, M., Namieśnik, J., 2012. PAH diagnostic ratios for the identification of pollution emission sources. Environ. Pollut. 162, 110-119.

Tuntawiroon, J., Mahidol, C., Navasumrit, P., Autrup, H., Ruchirawat, M., 2007. Increased health risk in Bangkok children exposed to polycyclic aromatic hydrocarbons from traffic-related sources. Carcinogenesis 28, 816-822.

Tuntawiroon, J., Mahidol, C., Navasumrit, P., Autrup, H., Ruchirawat, M., 2010. Increased health risk in Bangkok children exposed to polycyclic aromatic hydrocarbons from traffic-related sources. Carcinogenesis 28, 816-822.

U.S. Environmental Protection Agency (USEPA), 1989. Risk Assessment Guidance for Superfund, Vol. I: Human Health Evaluation Manual EPA/540/1-89/002 Office of Emergency and Remedial Response, Washington, DC.

U.S. Environmental Protection Agency (USEPA), 1990. Compendium of Methods for the Determination of Air Pollutants in Indoor Air. Atmospheric Research and Exposure Assessment Laboratory, Research Triangle Park, NC.

U.S. Environmental Protection Agency (USEPA), 2005. Guidelines for Carcinogen Risk Assessment, EPA/630/P-03/001F. US Environmental Protection Agency, Washington, D.C. http://www.epa.gov/raf/publications/pdfs/CANCER_GUIDELINES_FINAL_3-2505.pdf.

U.S. Environmental Protection Agency (USEPA), 2016. Risk-based concentration table. retrieved from http://www epa gov/reg3hwmd/risk/human/rb-concentration_table/ usersguide.htm accessed in February 2016.

Usenko, S.L., Massey Simonich, K.J., Haggeman, J.E., Schrlau, L., Geiser, D.H., Campbell, A.P.G., Landers, D.H., 2010. Sources and deposition of polycyclic aromatic hydrocarbons to western U.S. National Parks. Environ. Sci. Technol. 44, 4512-4518.

Vasilakos, C., Levi, N., Maggos, T., Hatzianestis, J., Michopoulos, J., Helmis, C., 2007. Gasparticle concentration and characterization of sources of PAHs in the atmosphere of a suburban area in Athens, Greece. J. Hazard. Mater. 140, 45-51.

Vedham, V., Verma, M., Mahabir, S., 2015. Early-life exposures to infectious agents and later cancer development. Cancer Medicine 4 (12), 1908-1922.

Wigle, D. , Arbuckle, T.E. Walker, M., Wade, M.G., Liu, S, Krewski, D. 2007. Environmental hazards: evidence for effects on child health. J. Toxicol. Environ. Health - Part B: Critical Reviews 10 (1-2), 3-39.

Wilson, N.K., Chuang, J.C., Lyu, C., Menton, R., Morgan, M.K., 2003. Aggregate exposures of nine preschool children to persistent organic pollutants at day care and at home. J. Expo. Anal. Environ. Epidemiol. 13, 187-202.

World Health Organization, 2000. Air Quality Guidelines for Europe. 2nd edition. WHO Regional Office for Europe, Copenhagen, Denmarkr.

World Health Organization (WHO), 1987. Air Quality Guidelines for Europe. WHO Regional Office for Europe, Copenhagen, Denmark

World Health Organization (WHO), 2010. WHO Guidelines for Indoor Air Quality: Selected Pollutants. Regional Office for Europe of the World Health Organization, Copenhagen, Denmark.

World Health Organization (WHO), 2013. State of the Science of Endocrine Disrupting Chemicals 2012. United Nations Environment Programme and the World Health Organization, Geneva.

Wu, F., Liu, X., Wang, W., Man, Y.B., Chan, C.Y., Liu, W., Tao, S., Wong, M.H., 2015. Characterization of particulate-bound PAHs in rural households using different types of domestic energy in Henan Province, China. Sci. Total Environ. 536, 840-846.

Zhang, W., Zhang, S., Wan, C., Yue, D., Ye, Y., Wang, X., 2008. Source diagnostics of polycyclic aromatic hydrocarbons in urban road runoff, dust, rain and canopy through fall. Environ. Pollut. 153, 594-601. 